\title{
Pathological a-synuclein recruits LRRK2 expressing pro-inflammatory monocytes to the brain
}

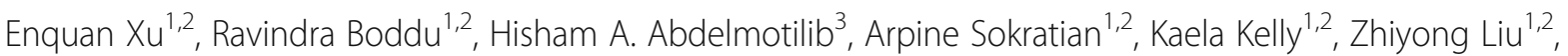
Nicole Bryant ${ }^{1,2}$, Sidhanth Chandra ${ }^{4}$, Samantha M. Carlisle ${ }^{5,6}$, Elliot J. Lefkowitz ${ }^{7}$, Ashley S. Harms ${ }^{8}$, Etty N. Benveniste ${ }^{9}$, Talene A. Yacoubian ${ }^{8}$, Laura A. Volpicelli-Daley ${ }^{8}$, David G. Standaert ${ }^{8}$ and Andrew B. West ${ }^{1,2^{*}}$ (D)

\begin{abstract}
Background: Leucine rich repeat kinase 2 (LRRK2) and SNCA are genetically linked to late-onset Parkinson's disease (PD). Aggregated a-synuclein pathologically defines PD. Recent studies identified elevated LRRK2 expression in proinflammatory CD16+ monocytes in idiopathic PD, as well as increased phosphorylation of the LRRK2 kinase substrate Rab10 in monocytes in some LRRK2 mutation carriers. Brain-engrafting pro-inflammatory monocytes have been implicated in dopaminergic neurodegeneration in PD models. Here we examine how a-synuclein and LRRK2 interact in monocytes and subsequent neuroinflammatory responses.
\end{abstract}

Methods: Human and mouse monocytes were differentiated to distinct transcriptional states resembling macrophages, dendritic cells, or microglia, and exposed to well-characterized human or mouse a-synuclein fibrils. LRRK2 expression and LRRK2-dependent Rab10 phosphorylation were measured with monoclonal antibodies, and myeloid cell responses to a-synuclein fibrils in R1441C-Lrrk2 knock-in mice or G2019S-Lrrk2 BAC mice were evaluated by flow cytometry. Chemotaxis assays were performed with monocyte-derived macrophages stimulated with a-synuclein fibrils and microglia in Boyden chambers.

Results: $a$-synuclein fibrils robustly stimulate LRRK2 and Rab10 phosphorylation in human and mouse macrophages and dendritic-like cells. In these cells, a-synuclein fibrils stimulate LRRK2 through JAK-STAT activation and intrinsic LRRK2 kinase activity in a feed-forward pathway that upregulates phosphorylated Rab10. In contrast, LRRK2 expression and Rab10 phosphorylation are both suppressed in microglia-like cells that are otherwise highly responsive to a-synuclein fibrils. Corroborating these results, LRRK2 expression in the brain parenchyma occurs in pro-inflammatory monocytes infiltrating from the periphery, distinct from brain-resident microglia. Mice expressing pathogenic LRRK2 mutations G2019S or R1441C have increased numbers of infiltrating pro-inflammatory monocytes in acute response to a-synuclein fibrils. In primary cultured macrophages, LRRK2 kinase inhibition dampens asynuclein fibril and microglia-stimulated chemotaxis.

\footnotetext{
* Correspondence: andrew.west@duke.edu

'Duke Center for Neurodegeneration Research, Duke University, Durham, NC 27710, USA

${ }^{2}$ Department of Pharmacology and Cancer Biology, Duke University, 3 Genome Court, Durham, NC 27710, USA

Full list of author information is available at the end of the article
} 
Conclusions: Pathologic a-synuclein activates LRRK2 expression and kinase activity in monocytes and induces their recruitment to the brain. These results predict that LRRK2 kinase inhibition may attenuate damaging proinflammatory monocyte responses in the brain.

Keywords: SNCA, PARK8, Monocyte extravasation, Neurodegeneration

\section{Background}

Rare missense pathogenic mutations in the leucine-rich repeat kinase 2 (LRRK2) gene that cause Parkinson's disease (PD) upregulate LRRK2 kinase activity [1, 2]. $L R R K 2$ is independently associated with PD through genome-wide association studies [3, 4], as well as with Crohn's disease and mycobacterium infection susceptibility [5]. Common genetic variation in the LRRK2 locus that associates with PD risk links to the quantitative expression of pro-inflammatory genes in monocytes [6]. In humans, LRRK2 is principally expressed in blood (gtexportal.org), and LRRK2 protein levels are elevated in classical (e.g., pro-inflammatory) monocyte populations as measured in idiopathic PD patients (lacking pathogenic LRRK2 mutations) in two different study populations in Europe and the United States [7, 8]. The cause of upregulated LRRK2 levels in monocytes in PD is not known, and LRRK2 transcriptional regulation and control of downstream LRRK2 kinase activity is not well understood in monocytes.

In monocyte-derived macrophages, LRRK2 expression and activity upregulates chemotaxis and AKT-dependent chemokine receptor signaling through phosphorylation of the LRRK2-kinase substrate Rab10 [9-11]. PDassociated mutations in $L R R K 2$, as well as mutated VPS35, upregulate LRRK2-dependent Rab10 phosphorylation in monocytes [12]. Considering the potential role for LRRK2 in chemotaxis, brain-engrafting monocytederived macrophages have been previously implicated in the destruction of dopaminergic neurons in the rAAV2$\alpha$-synuclein expression model and the toll-like receptor (TLR)-4 agonist lipopolysaccharide (LPS) model [13, 14]. In the rat brain, Lrrk2 protein colocalizes inside activated $\mathrm{CD} 68+$ myeloid cells of unknown origins, recruited in response to rAAV2- $\alpha$-synuclein expression or intracranial injections of LPS [15]. In the pre-formed $\alpha$ synuclein fibril model, immune cell recruitment to the brain may occur early after fibril injection, persists for months, and causes a notable systemic upregulation of pro-inflammatory cells $[16,17]$. In both the AAV and fibril models, owing to the broad overlap and dynamic nature of protein and lipid markers used to identify different types of myeloid cells, the identity of the specific innate immune cell compartments where LRRK2 might exert intrinsic function to potentially modify neuroinflammatory phenotypes in disease has not been clear.
The principle sources of innate immune cells in both human and rodent brains include abundant resident microglia as well as non-resident macrophages found in the perivasculature and meningeal spaces [18]. Based on past studies that demonstrate robust LRRK2 effects on microglial responses both in cell culture and in mouse models [19-22], it is widely presumed that intrinsic LRRK2 expression in microglia drives immunological phenotypes in the brain. Potentially at odds with this assumption, Lrrk2 expression is relatively low or sometimes undetectable in the sizable population of sentinel microglia in rodent or human brain as assessed by recent single-cell sequencing efforts [23, 24] or through immunohistochemistry $[25,26]$. However, other studies have convincingly demonstrated LRRK2 function in isolated microglia cells in culture, potentially functioning through NFATc2 and toll-receptor signaling pathways $[19,21,27,28]$.

Here, we seek to resolve the different types of immune cells harboring LRRK2 protein expression and Rab10 phosphorylation in response to PD-associated aggregated $\alpha$-synuclein. Using a powerful comparative strategy developed by Ryan et al. to polarize human bloodderived monocytes to distinct transcriptional states [29], in complement with observations in vivo in mouse models, our results show that LRRK2 protein levels, kinase activity, and induction by pathological $\alpha$-synuclein are largely restricted to monocyte-derived macrophages, distinct from microglia. In mice, LRRK2-kinase activating mutations G2019S and R1441C tend to enhance the recruitment of pro-inflammatory monocyte-derived macrophages to the brain, in immune cells that express the highest levels of LRRK2 protein, consistent with a role for LRRK2 in chemotaxis observed in cell culture models. These results provide evidence that LRRK2 may drive neuroinflammatory responses in disease in part through promoting the recruitment of pro-inflammatory monocytes to the brain.

\section{Methods}

\section{Human cell cultures}

Blood mononuclear cells were isolated from venous blood draws from healthy volunteers. Healthy volunteers in this study (age range 24-34) did not have a family history of PD, did not report ancestries known to be enriched with pathogenic LRRK2 mutations, and were not taking anti-inflammatory medications. Blood draws 
were processed with SepMate (Fisher) tubes and Lymphoprep (Stemcell Tech) tubes according to manufacturer's recommendations and previous reports [29]. EasySep Negative Selection Human Monocyte Enrichment kits (Stemcell Tech), without CD16 depletion, were used according to manufacturer's recommendations. Macrophage cells (i.e., monocyte-derived macrophages, MDMs) were cultured in DMEM (Invitrogen) supplemented with Glutamax (Invitrogen), 10\% fetal bovine serum (FBS), 1\% penicillin/streptomycin (Invitrogen), Fungizone $\left(2.5 \mathrm{~g} \mathrm{~mL}^{-1}\right)$, and human MCSF (20 ng $\left.\mathrm{mL}^{-1}\right)$. For dendritic-like cells and microglia-like cells [29], media included RPMI-1640 (Invitrogen), Glutamax (Life-Technologies), 1\% penicillin/streptomycin (Invitrogen), and Fungizone $\left(2.5 \mathrm{~g} \mathrm{~mL}^{-1}\right.$; Life Technologies). Dendritic-like cells were further supplemented with 10\% FBS (Bio-Techne) and human GMCSF (5 ng $\left.\mathrm{mL}^{-1}\right)$. Microglia-like cells were supplemented with MCSF (10 ng mL-1), GMCSF (10 ng $\mathrm{mL}^{-1}$ ), NGF- $\beta$ (10 ng $\left.\mathrm{mL}^{-1}\right)$, CCL2 (100 ng $\left.\mathrm{mL}^{-1}\right)$, and IL-34 (100 ng $\left.\mathrm{mL}^{-1}\right)$. All cytokines were purchased from PeproTech. All cells were cultured for at least 1 week before experiments. As indicated, some cells were activated with LPS (100 ng $\mathrm{mL}^{-1}$ triple-purified from E. coli strain O55:B5, InvivoGen) for $48 \mathrm{~h}$. Alternativelyactivated cells, as indicated, were generated with the addition of $20 \mathrm{ng} \mathrm{mL}^{-1} \mathrm{IL}-4$ and IL-13 for $48 \mathrm{~h}$.

\section{Mice and mouse cell culture}

Lrrk2 R1441C-KI (JAX Stock \# 009346), Lrrk2 WT-BAC transgenic mice (JAX stock \#012466), Lrrk2 G2019SBAC (JAX stock \#012467), Lrrk2 knock-out (KO) and C57BL/6 J WT (nTg) control mice (JAX stock \#000664) were obtained from Jackson Laboratories. Mice were genotyped according to published protocols by the depositing investigators, including quantitative PCR protocols used for measuring BAC copy numbers through the course of the project. Mouse bone marrow-derived cells were procured from 3 to 5 months-old mice. Bone marrow cells were flushed with ice-cold PBS and transferred through $70 \mu \mathrm{m}$ nylon cell strainers (Millipore). Cells were centrifuged at $450 \mathrm{xg}$ for $10 \mathrm{~min}$ at $4{ }^{\circ} \mathrm{C}$ and incubated with red blood cell lysis buffer (Invitrogen) for 30 s. Cells were dissociated and cultured with DMEM media supplemented with $10 \%$ FBS and $20 \mathrm{ng} \mathrm{mL}^{-1}$ mouse MCSF (PeproTech). The immortalized mouse microglial cell line BV-2 (ATCC CRL-2469) was cultured as previously described [30].

For chemotaxis experiments, primary mouse bone marrow-derived macrophages were applied to the upper chamber of Transwell dishes (Corning Costar, CLS3464), and fibril-conditioned media was added into the lower chamber at a 1 to $10(\mathrm{w} / \mathrm{v})$ ratio. Two hours post-conditioned media addition, non-mobile cells in the upper chamber were removed, washed, and the upper surface layer swabbed with cotton. The migrated cells at the bottom surface of the Transwell membrane were fixed with $4 \%$ paraformaldehyde and stained with DAPI for automated counting on a Keyence BZ-X800 microscope.

\section{Recombinant a-synuclein}

Bacterial expression plasmids encoding mouse and human WT- $\alpha$-synuclein in the inducible pRK172 backbone were transformed into BL21-CodonPlus (DE3) cells (Clontech). Cell pellets were lysed in $0.75 \mathrm{M} \mathrm{NaCl}, 10$ mM Tris- $\mathrm{HCl}, \mathrm{pH} 7.6,1 \mathrm{mM}$ EDTA, $1 \mathrm{mM}$ PMSF and sonicated at $70 \%$ power (Fisher500 Dismembrator) for 1 $\mathrm{min}$, and tubes were placed in boiling water for $15 \mathrm{~min}$. Centrifuged samples were dialyzed against $10 \mathrm{mM}$ Tris (pH 7.6) with $50 \mathrm{mM} \mathrm{NaCl}, 1 \mathrm{mM}$ EDTA, $1 \mathrm{mM}$ PMSF. The suspension was passed through a HiPrep Q HP 16/ 10 column (GE Healthcare) on an ÄKTA pure protein purification system (Cytiva) with a running buffer composed of $10 \mathrm{mM}$ Tris $\mathrm{pH} 7.6,25 \mathrm{mM} \mathrm{NaCl}$, and eluted with a linear gradient application of high-salt buffer (10 $\mathrm{mM}$ Tris, $1 \mathrm{M} \mathrm{NaCl} \mathrm{pH}$ 7.6). Samples containing singleband profiles of $\alpha$-synuclein were identified by Coomassie staining and further dialyzed and concentrated. Monomer protein passed through three rounds of endotoxin removal (Endotoxin removal kit, GenScript) to reach a low level of $<0.1$ E.U. $\mathrm{mg}^{-1}$, with endotoxin levels determined using a LAL chromogenic endotoxin quantification kit (GenScript). Mouse or human $\alpha$ synuclein fibrils were prepared through incubation of 7 $\mathrm{mg} \mathrm{mL}^{-1} \alpha$-synuclein monomer in phosphate-buffer saline for 7 days at $37^{\circ} \mathrm{C}$ with constant agitation. Fibrils were washed and subjected to a second round of amplification as described [31]. Final sonicated preparations were measured by dynamic light scattering on a Titan DynaPro (Wyatt Technology), with molecular weights of terminal particles estimated from $\mathrm{A}_{280}$, intensity, and mass distributions, as described [31].

\section{Intracranial stereotaxic injections}

Consistent with past studies [32], $5 \mu \mathrm{g} \quad \mathrm{mL}^{-1}$ preparations of human $\alpha$-synuclein fibrils or monomer $(2 \mu \mathrm{L}$ per site) were injected into $\sim 8-16$ weeks old mice. 30-gauge needles (Hamilton) with a $110^{\circ}$ bevel were used for injections with solutions infused using an automated stereotaxic injector (Stoelting) at a flow rate of $0.25 \mu \mathrm{L}$ per min with the bevel of the needle facing medially according to stereotaxic coordinates with respect to Bregma (AP $-3.1 \mathrm{~mm}, \mathrm{ML} \pm 1.5$, DV $-4.6 \mathrm{~mm}$ ). At the indicated time of sacrifice, mice were transcardially perfused with cold PBS ( $\mathrm{pH}$ 7.4) and brains removed and dissected for flow cytometry. 


\section{Flow cytometry}

Whole brains were procured from mice perfused with cold PBS and digested with $1 \mathrm{mg} \mathrm{mL} \mathrm{mL}^{-1}$ collagenase IV (Sigma) with $20 \mu \mathrm{g} \mathrm{mL} \mathrm{mL}^{-1}$ DNAse I (Sigma) diluted in RPMI-1640 with 10\% FBS, 1\% L-glutamine, and 1\% penicillin-streptomycin (Invitrogen) as described [13, 33]. Mononuclear cells were isolated with a 30/70\% Percoll gradient and blocked with $\mathrm{Fcy}$ receptor antibody clone 2.4G2. Leukocytes from the spleen were isolated as previously described [34]. Anesthetized mice were perfused with cold PBS and minced spleens were digested with Liberase DL (Roche Diagnostics, Inc.) in DMEM (30 min at $37^{\circ} \mathrm{C}$ with shaking). Single-cell suspension from splenic tissues were prepared by passing through $18 \mathrm{Ga}$. and $20 \mathrm{Ga}$. needles followed by passage through a 40- $\mu$ m nylon filter. An Ammonium-ChloridePotassium (ACK) lysis buffer (Invitrogen) was used for lysing red blood cells. For some experiments, mouse midbrains were dissected from whole brain dissections from a $2 \mathrm{~mm}$-thick coronal slice, with the midbrain carefully removed, minced, and tissue pieces triturated and digested in $1 \mathrm{mg} \mathrm{mL} \mathrm{m}^{-1}$ collagenase IV with $20 \mu \mathrm{g}$ $\mathrm{mL}^{-1}$ DNAse I diluted in HBSS (Invitrogen) for $35 \mathrm{~min}$ at $37^{\circ} \mathrm{C}$ in a water bath with gentle intermittent shaking. The digestion was halted with incubation in PBS, $2 \mathrm{mM}$ EDTA, and $1 \% \mathrm{w} / \mathrm{v}$ bovine-serum albumin (Sigma) on ice.

Antibody clones were obtained from eBioscience, BioLegend, BD Pharm, Abcam and Life Technologies and included CD45 brilliant violet (BV) 650 (clone 104); CD45 BV650 (clone 30-F11); CD45 eFluor450 (clone 30F11); CD11b eVolve 605 (clone M1/70); Ly6G allophycocyanin-conjugated (APC) (Gr-1, clone 1A8); Ly6C eFluor(eF)450 (clone HK1.4); CD11b BV605 (clone M1/70); CD11b phycoerythrin (PE) (clone M1/70); MHCII FITC (I-A/I-E); CD4 PE-Cyanine7 (clone GK1.5); CD8a APC-Cyanine7 (clone 53-6.7) and rabbit monoclonal [MJFF2 (c41-2)] anti-LRRK2 (AlexaFluor 647), in addition to 7-aminoactinomycin D (7-AAD) for live-dead staining and fixable viability dye (Live/Dead Fixable Dead Cell Stain Kit, Near IR). All cell suspensions were analyzed on an Attune NxT instrument (acoustic-assisted hydrodynamic focusing cytometer; Thermo Fisher Scientific) and all results/cytometer acquired data were analyzed using FlowJo 10.7 software (BD Company).

Flow cytometry for detecting intracellular LRRK2 in mouse cells was accomplished according to Bliederhaeuser and Cook with several modifications $[7,8]$. The twostep protocol for intracellular (cytoplasmic) proteins was performed with the Intracellular Fixation and Permeabilization Buffer set (eBioscience) according to manufacturer's recommendations with the rabbit monoclonal [MJFF2 (c41-2)] anti-LRRK2 antibody conjugated to Alexa Fluor 647. Alexa Fluor647 median fluorescence intensity (MFI) signals from Lrrk2 $\mathrm{KO}$ mice were subtracted from each cell population processed in parallel. All experiments were analyzed with FlowJo 10.7 software (BD Company).

\section{Immunoblotting and immunofluorescence}

Tissues were collected following transcardial perfusion with cold PBS, and tissues (or cells in culture) were homogenized with probe-tip sonication in RIPA lysis buffer containing $50 \mathrm{mM}$ Tris (pH 7.4), $150 \mathrm{mM} \mathrm{NaCl}, 1 \%$ Triton, and $0.1 \%$ SDS supplemented with $1 \mathrm{x}$ Complete protease and PhosStop inhibitor tablets (Roche). Homogenates were centrifuged and soluble supernatants analyzed using the BCA assay (Pierce). Lysates were analyzed on $4-20 \%$ gradient mini-PROTEAN TGX stainfree gels (BioRad) and transferred to Immobilon-FL PVDF membrane (Millipore), followed by immunoblotting with the indicated primary and secondary antibodies. The following antibodies were used: N241/34 anti-LRRK2 (Antibodies Inc), phospho-T73-Rab10 (MJFR21, Abcam), total Rab10 antibody (MJF-R23, Abcam), pY701-Stat1 (Tyr701, D4A7, CST), total Stat1 (42H3, CST), loading control Hsc70 (Cell Signaling), donkey anti-mouse 680LT (LiCor), and goat anti-rabbit HRP (Jackson Immuno). For relative quantifications, the mean of the selected experimental control group is defined as the reference value after normalization to $\beta$-actin levels in the same lane. All signals were captured digitally using a Chemidoc MP Imaging System (BioRad). Quantifications were performed using Image Lab 6.0.1 software (BioRad).

Confocal images of immunostained cells, prepared as previously described [9], were captured either on a Zeiss 880 AiryScan confocal microscope, and brightfield images were captured on an Olympus BX61 microscope. Confocal images were acquired using Zen software (Zeiss), with contrast and color balance adjusted equally across all images in the group in Adobe Illustrator. Original images are available upon request from the Authors.

\section{Small molecule inhibitors}

All compounds were $>98.5 \%$ pure as assessed by LC/MS and NMR analysis. The small molecule LRRK2 inhibitor MLi2 was synthesized for this study using the previously described strategy by Pharmaron, Inc. [35]. AZD1480 is an ATP-competitive JAK1/2 inhibitor with an $\mathrm{IC}_{50}$ of $0.26 \mathrm{nM}$ (Selleckchem, Inc). Tofacitinib citrate (CP690550, Selleckchem, Inc) is an inhibitor for JAKs with an $\mathrm{IC}_{50}$ of $1 \mathrm{nM}, 20 \mathrm{nM}$ and $112 \mathrm{nM} \mathrm{IC} 50$ against JAK3, JAK2, and JAK1, respectively. Ruxolitinib (Selleckchem, Inc) is a selective JAK1/2 inhibitor with an $\mathrm{IC}_{50}$ of 3.3 $\mathrm{nM} / 2.8 \mathrm{nM}$, respectively. 


\section{Transcriptomic analysis}

Nucleic acids were collected with TRIzol (Invitrogen) reagent. Total RNA was extracted with the Qiagen RNeasy Plus Universal mini kit following manufacturer's instructions (Qiagen, Germany), and extracted RNA samples were quantified using Qubit 2.0 Fluorometer (Life Technologies, USA) and RNA integrity $>0.9$ was verified with an Agilent TapeStation 4200 (Agilent Technologies, USA). Libraries were prepared with the NEBNext Ultra RNA Library Prep Kit according to manufacturer's instructions (NEB, Inc.). Sequencing libraries were pooled and clustered on a single lane of a flow cell on an Illumina HiSeq instrument according to manufacturer's instructions. Samples were sequenced using a $2 \times 150 \mathrm{bp}$ paired end configuration, with image analysis and base calling conducted with HiSeq Control Software (Illumina). Raw sequence data files were converted to fastq files and de-multiplexed using bcl2fastq 2.17 software. One mismatch was allowed for index sequence identification. Quality of the sequencing reads were interrogated with FastQC (version 0.11.5), and aligned with STAR aligner (version 2.5.2) using basic 2-pass mapping, with all 1st pass junctions inserted into the genome indices to GENCODE release 31 GRCh38.p12 genome using the GENCODE v31 transcript annotation, with an average of $94 \%$ of reads aligning, giving an average of 46.8 million uniquely aligned reads per sample presented in this study. Analysis and visualization of the resulting data were performed using $\mathrm{R}$ version 4.0.0 software. Aligned reads were quantified using the GenomicAlignments package (Bioconductor) with the "IntersectionStrict" setting. Principal component analysis (PCA) in the $\mathrm{R}$ environment was conducted by singular value decomposition of the centered data matrix on the most 1000 variable genes in the entire dataset. The loadings of the first ( $\mathrm{x}$-axis) and second (y-axis) principal components were plotted.

\section{Other statistics and rigor}

Experiments described were independently repeated at least three times and bar graphs show mean values with SEM error bars. Data points for in vivo experiments represent individual mice. All raw data are available as Supplemental Information. Uncropped western blot images collected from the ChemiDoc analysis are available as Supplemental Information. Group statistical analyses were conducted using Graphpad Prism 8.0 software. Continuous data with more than two independent groups were evaluated for significance using a one- or two-factor ANOVA, with the indicated post-hoc test. Two-tailed, unpaired $t$-tests were used to determine the significance between two groups. All tests were performed using a significance level of $\alpha=0.05$ with $95 \%$ confidence. All groups are presented with mean values and error bars representing SEM. Samples were processed without respect to group assignment, and final data were curated before sample assignment to groups. Mice were randomized with respect to genotype and sex across all experiments.

\section{Results \\ a-synuclein fibrils induce LRRK2 expression and Rab10 phosphorylation in mouse and human monocyte-derived macrophages}

Recent studies suggest neuronal $\alpha$-synuclein inclusions are composed in part of $\alpha$-synuclein fibrils intermixed with membranous organelles [36, 37]. Recombinant $\alpha$ synuclein fibrils, but not recombinant monomeric or oligomeric protein, potently stimulate innate immune cells $[13,17]$. To determine whether $\alpha$-synuclein fibrils might affect LRRK2 expression and kinase activity in immune cells, assessed inpart through measures of the LRRK2 kinase substrate Rab10 [9], cultured mouse bone-marrow derived macrophages were combined with low concentrations of mouse $\alpha$-synuclein fibrils $(\sim 0.7$ $\mathrm{nM})$, or the equivalent amount of monomeric $\alpha$ synuclein protein $(\sim 70 \mathrm{nM})$ as a control (Fig. 1A-C and Supplemental Fig. 1). In these preparations of recombinant $\alpha$-synuclein, contaminating endotoxin was measured as less than 0.001 endotoxin units (E.U.) per mL. Previously, we used this concentration of fibrils as one of the lowest concentrations required to seed new inclusions in neurons in culture [38, 39]. Fibrils, but not monomeric protein, induced Lrrk2 protein expression $(\sim 2.5$ fold, Fig. 1D, E) as well as increased pT73-Rab10 levels $(\sim 3$ fold, Fig. 1F). Treatment with the LRRK2 kinase inhibitor, MLi2 $(100 \mathrm{nM})$, interrupted $\alpha$-synuclein fibrilinduction of total Lrrk2 protein expression and reduced pT73-Rab10 levels (Fig. 1D-F). The ATP-competitive MLi2 molecule is selective and not known to interact with other kinases or proteins at this concentration [35, $40,41]$. In macrophages without $\alpha$-synuclein fibril stimulation, MLi2 exposure did not reduce basal levels of total Lrrk2 protein, or Lrrk2 levels after incubation with $\alpha$-synuclein monomers (Fig. 1D-F). Thus, the MLi2 inhibitor itself does not reduce Lrrk2 levels in macrophages, but prevents $\alpha$-synuclein-fibril induced stimulation of Lrrk2.

In primary human monocyte-derived macrophages cultured from human healthy controls, the application of human $\alpha$-synuclein fibrils, prepared in a similar manner to the mouse $\alpha$-synuclein fibrils [31], led to a similar induction of LRRK2 protein expression as observed in mouse macrophages $(\sim 2$ fold, Fig. $1 \mathrm{G}, \mathrm{H})$, as well as increased pT73-Rab10 levels ( 3.8 fold, Fig. 1I). Consistently, LRRK2 kinase inhibition, through MLi2 exposure, appeared equally effective against both mouse and human LRRK2, blocking $\alpha$-synuclein-induced upregulation 


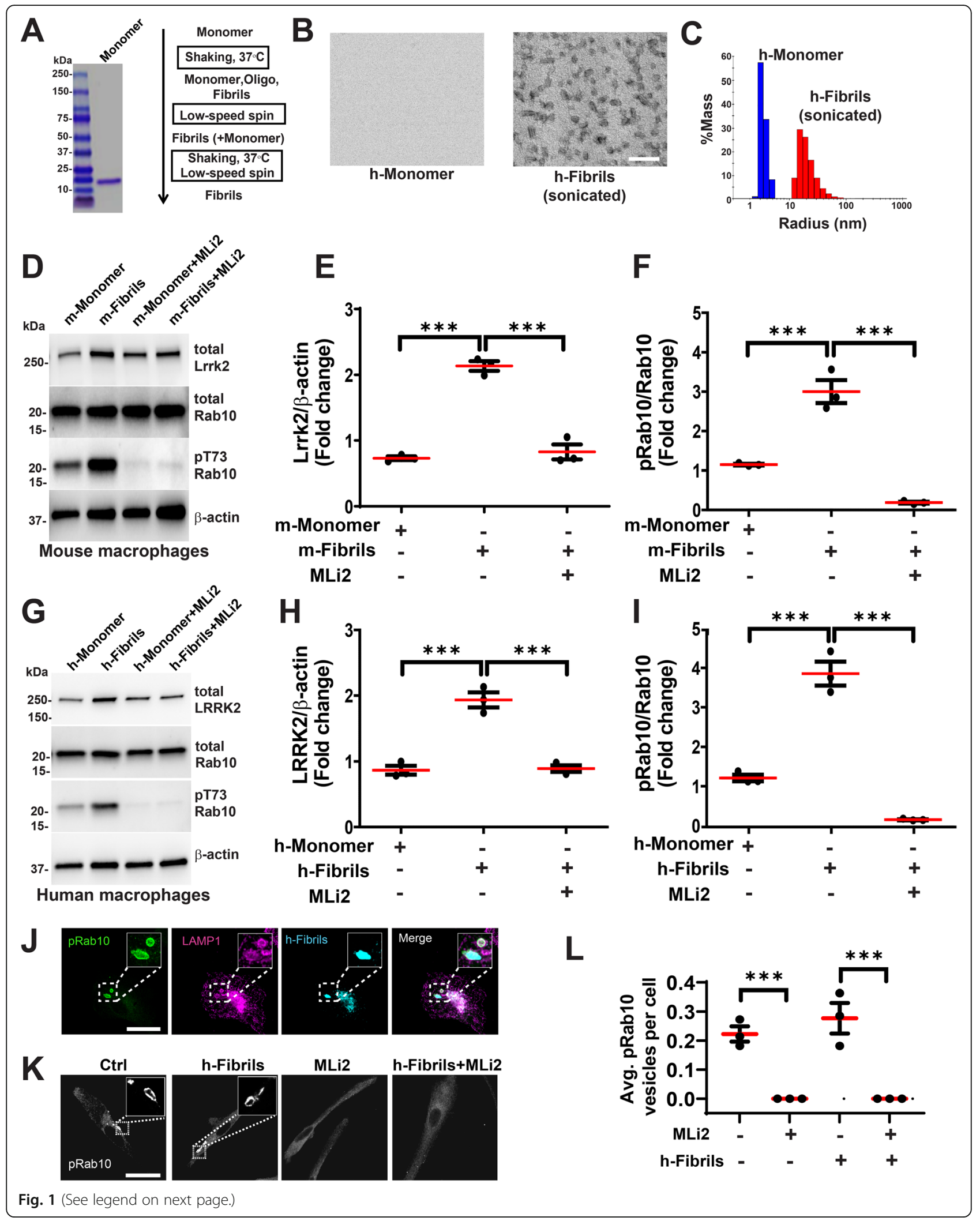


(See figure on previous page.)

Fig. 1 a-Synuclein fibrils induce Lrrk2/LRRK2 in monocyte-derived macrophages. A a-Synuclein purification and short-rod fibril construction. Preparations were verified to have low or no detectable endotoxins (see Methods). Additional quality control data are presented in Supplemental Fig. 1. B Electron-microscopy photomicrographs of representative human a-synuclein monomers and sonicated a-synuclein fibrils. Scale bar is $100 \mathrm{~nm}$. C Representative dynamic light-scattering characterization of matched monomer and fibril preparations. D Representative immunoblots of mouse (male nTg C57BI/6 J) bone-marrow derived macrophages, expanded with MCSF, exposed to mouse fibrils $\left(\sim 0.7 \mathrm{nM}, 1 \mu \mathrm{g} \mathrm{mL}{ }^{-1}\right.$, see Methods), or mouse monomer ( 70 nM, $\left.1 \mu \mathrm{g} \mathrm{mL} \mathrm{mL}^{-1}\right)$, with or without the LRRK2 inhibitor MLi2 (100 nM). Results from the quantification of three independent experiments for $\mathbf{E}$ Lrrk2 protein and F pT73-Rab10 levels (ratio of pT73-Rab10 to total Rab10 protein). G Representative immunoblots of human (two healthy males and one healthy female, age range 24 to 34 years old) monocyte-derived macrophages (MCSF expanded) with quantification from three independent experiments for $\mathbf{H}$ LRRK2 protein and I pT73-Rab10 levels. J Representative confocal images of pT73Rab10, LAMP1, and pHRodo-labeled human a-synuclein fibrils $\left(1 \mu \mathrm{g} \mathrm{mL}^{-1}\right)$ in human macrophages, $\mathbf{K}$ with or without MLi2 (100 nM). Scale bars are $50 \mu \mathrm{m}$. L Quantification of the average number of pT73-Rab10 vesicles per cell. Each dot represents the mean vesicles per cell of images procured from three independent experiments, with $\sim 75 \%$ of cells in control wells not showing any pT73-Rab10 vesicles, consistent with past observations in macrophages [9]. All data are group means \pm SEM from $n=3$ biologically independent experiments. Significance is determined by one-way ANOVA with Tukey's post hoc test and ${ }^{* * *} p<0.001$

of LRRK2 protein expression and pT73-Rab10 levels (Fig. 1G-I). Previously, we identified that pT73-Rab10 protein in macrophages specifically localized to signaling endosomes, often co-positive for CCR5-receptors and LAMP1 [9]. In the context of $\alpha$-synuclein fibril exposure, pT73-Rab10 vesicles were similarly LAMP1 positive and appeared co-positive with exogenously-applied labeled (pH-Rhodo) $\alpha$-synuclein fibrils (Fig. 1J). MLi2 completely ablated the detection of pT73-Rab10 vesicles visualized by immunofluorescence (Fig. 1K). While levels of pT73-Rab10 increased in lysates after fibril administration as measured by immunoblotting, $\alpha$-synuclein fibril administration did not have a noticeable effect on increasing the total number of pT73-Rab10 vesicles per cell (Fig. 1L). These results suggest the amount of Rab10 phosphorylated on vesicles increases with fibrils of either mouse or human origin, is dependent on LRRK2 kinase activity, and occurs without an increase in the overt number of vesicles in the macrophage cytoplasm.

\section{a-synuclein fibrils activate LRRK2 through JAK-STAT signaling}

Past studies suggest that aggregated $\alpha$-synuclein may stimulate TLR4 signaling pathways in innate immune cells $[42,43]$. We and others have previously implicated LRRK2 in TLR4 signaling [19, 26, 44]. In human primary monocyte-derived macrophages, we found that the application of a potent TLR4 inhibitor failed to block $\alpha$ synuclein fibril-induction of LRRK2 protein expression and Rab10 phosphorylation (Supplemental Fig. 2). To search for other pathways that might regulate LRRK2 induction, a comparative analysis of several transcriptomes of human primary monocyte-derived macrophages treated with $\alpha$-synuclein fibrils highlighted components of JAK-STAT signaling including STAT1 and JAK2 mRNA together with LRRK2 mRNA (Supplemental Fig. 3). We hypothesized that $\alpha$-synuclein fibrils might stimulate the JAK-STAT pathway to promote LRRK2 expression. To test this possibility, we treated cultures with three structurally distinct JAK inhibitors $1 \mathrm{~h}$ prior to fibril administration and measured subsequent LRRK2 and Rab10 phosphorylation changes (Fig. 2). While ruxolitinib and AZD1480 primarily target JAK1/2 at low nanomolar concentrations $[45,46]$, tofacitinib-citrate primarily targets JAK2/3 $[47,48]$. After fibril treatment, LRRK2 levels and activity were upregulated early and sustained consistently to $72 \mathrm{~h}$ post-fibril treatment in vehicle-only conditions (Fig. 2A-K). All three inhibitors effectively reduced $\alpha$-synuclein fibril-induced LRRK2 expression levels and pT73-Rab10 levels, without apparent morphological changes to the cells (Fig. 2B). These results indicate that the upregulation of JAK-STAT signaling caused by fibril exposure is necessary for the upregulation of LRRK2 expression and subsequent Rab10 phosphorylation.

\section{Effect of innate immune cell differentiation on a- synuclein fibril induction of LRRK2}

LRRK2 expression and function has been described in a variety of immune cells including neutrophils and monocytes $[8,49]$, and by immunohistochemistry in CD68+ macrophage subsets of unspecified origins that can accumulate in the rat brain in the context of LPS and rAAV$\alpha$-synuclein insults [15]. According to Ryan et al., naive human blood monocytes procured through negative selection can be polarized to functionally and transcriptionally resemble different types of innate immune cells including microglia-like (MDMi) cells [29]. CD14+/ $\mathrm{CD} 16+$ monocytes were isolated from healthy volunteers and differentiated to resemble dendritic-like (GMCSF) cells, macrophage-like (MCSF) cells, and MDMi cells. Resultant cell populations differentiated for ten-days in culture (Fig. 3A) were morphologically similar in presentation (Fig. 3B) but transcriptionally distinct (Fig. 3C). Functional differentiation to these different states was characterized by CD163 expression that was high in MCSF cells, high CCR2 expression in GMCSF cells, and high P2RY12 in MDMi cells (Fig. 3D, with fluorescence 


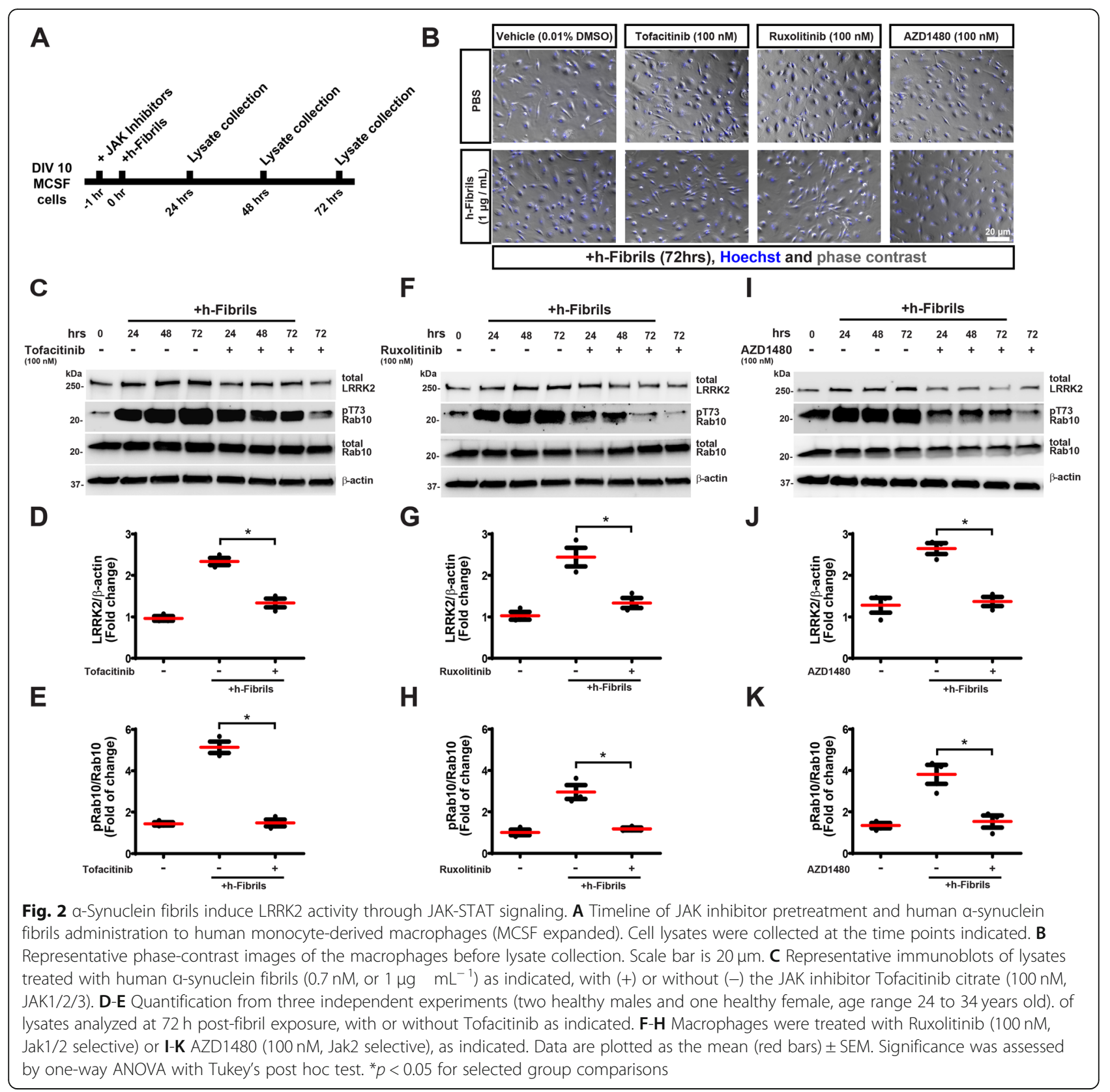

intensities quantified in Supplemental Fig. 4). HLA-DR is a canonical pro-inflammatory surface marker robustly detected in PD brains [50], and fibrils stimulated HLADR $\alpha$ expression in both MCSF and MDMi cells (Fig. 3E, F). Similar to MCSF cells, GMCSF cells demonstrated upregulated LRRK2 and pT73-Rab10 levels after $\alpha$ synuclein fibril exposures that could be completely mitigated by concurrent AZD1480 treatment (Fig. 3E, F). However, in MDMi cells, fibrils failed to stimulate LRRK2 or Rab10 phosphorylation. In contrast, MDMi cells robustly responded to the fibrils with IL-6 cytokine secretion to a similar (or slightly increased) extent as compared to MCSF macrophage cells (Fig. 3I). These results suggest that microglia have low levels of LRRK2 kinase activity in response to $\alpha$-synuclein fibril exposures, in contrast to macrophages and dendritic-like cells that show strong induction of LRRK2 kinase activity in response to fibrils.

\section{a-synuclein fibrils recruit Lrrk2-monocytes to the brain}

In human brain tissue, activated antigen-presenting innate immune cells are often nearby neuronal $\alpha$ synuclein inclusions [51]. Both $\alpha$-synuclein overexpression or intracranial LPS injection causes the accumulation of CD68-positive macrophages in the brain that may originate from resident innate immune cells 

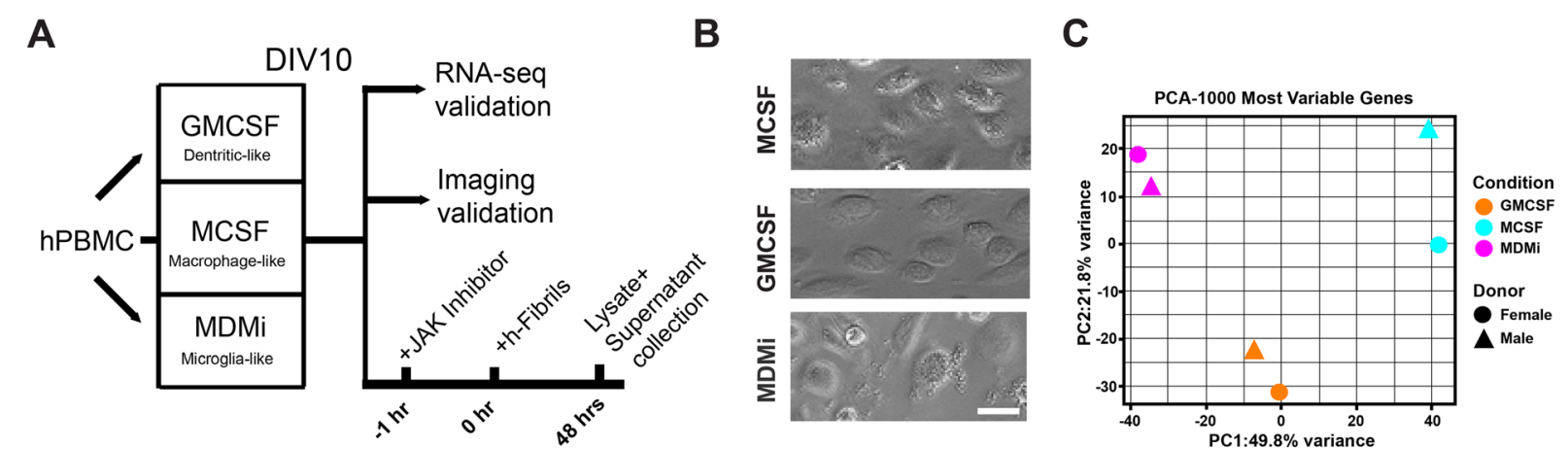

D
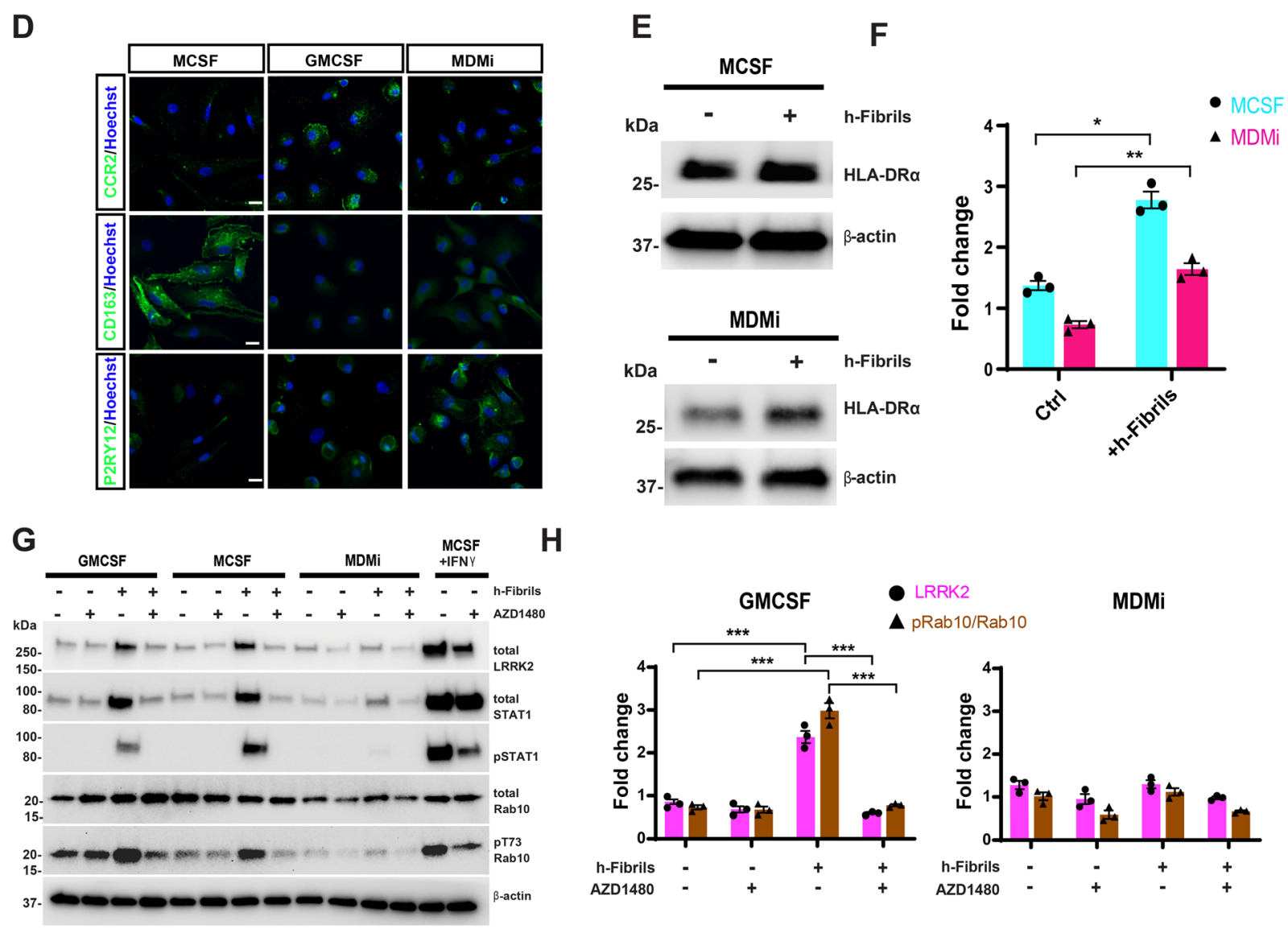

I

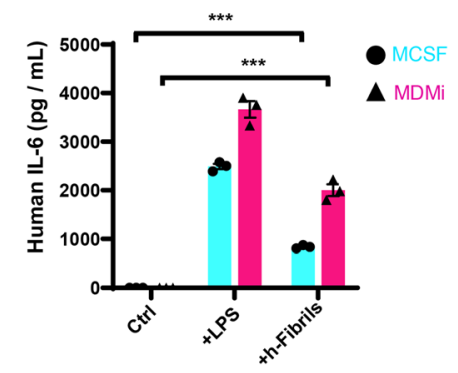

Fig. 3 (See legend on next page.) 
(See figure on previous page.)

Fig. 3 Microglia-like polarization suppresses a-synuclein fibril induction of LRRK2 expression and activity. A Flow chart of PBMC differentiation into three monocyte lineages, with validation (mRNA-seq and fluorescent imaging of canonical markers), and treatment timeline. The JAK inhibitor (AZD1480, $100 \mathrm{nM}$ ) was applied $1 \mathrm{~h}$ before human a-synuclein fibrils $\left(1 \mu \mathrm{g} \mathrm{mL}{ }^{-1}\right)$, and cell lysates were collected $48 \mathrm{~h}$ later. B Representative phase-contrast images of human monocytes differentiated to dendritic-like cells (GMCSF), macrophage-like cells (MCSF), and microglia-like cells (MDMi). C Principal component analysis of the resultant transcriptomes (circle: female, triangle: male, orange: GMCSF, cyan: MCSF, pink: MDMi). D Representative immunostaining of canonical markers for the different lineages (CD163 for macrophages MCSF; CCR2 for dendritic cells GMCSF; and P2RY12 for microglia cells MDMi). Quantification presented in Supplemental Fig. 4. Scale bars are $10 \mu \mathrm{m}$. E Representative immunoblots, and $\mathbf{F}$ quantification for HLA-DRa upregulation, $24 \mathrm{~h}$ after human a-synuclein fibril treatment in both macrophages and microglia-like (MDMi) cells. G Representative immunoblots of lysates from cells treated with or without human a-synuclein fibrils $(1 \mu \mathrm{g}$ $\mathrm{mL}^{-1}, 48 \mathrm{~h}$ ), with or without the JAK2 inhibitor AZD1480 (100 nM). IFNy (20 $\mathrm{ng} \mathrm{mL} \mathrm{mL}^{-1}$ ) treated macrophages (MCSF) were used as a positive control for LRRK2 upregulation and functional activation in the different cell lineages. H Quantification of total LRRK2 and the ratio of pT73-Rab10 to total Rab10 in dendritic (GMCSF) and microglia-like cells (MDMi). I From cell culture media, human IL-6 levels were measured by ELISA in untreated cells (control), cells treated with LPS (100 ng $\mathrm{mL}^{-1}, 24 \mathrm{~h}$ ), or human a-synuclein fibrils $\left(1 \mu \mathrm{g} \mathrm{mL} \mathrm{mL}^{-1}, 24 \mathrm{~h}\right)$. All data show mean \pm SEM for $n=3$ independent experiments, except for transcriptional analysis in panel $\mathrm{C}$ that includes a male and female pair (healthy controls, age range 24 to 34 years old). Significance is assessed by one-way ANOVA with Tukey's post hoc test where ${ }^{*} p<0.01,{ }^{* *} p<0.01,{ }^{* * *} p<0.001$

(e.g., microglia) or infiltrating bone-marrow-derived cells (e.g., monocytes) $[15,19,52]$. To track expression in immune cells isolated directly from the perfused mouse brain, we established a flow cytometry protocol with previously described Lrrk2-targeting rabbit monoclonal antibodies [25]. For optimization, initial Lrrk2 intracellular flow cytometry experiments were performed with spleen tissues to identify staining conditions compatible with Lrrk2 protein detection in monocytes, with the Lrrk2 knockout mouse strain as a control (Fig. 4A). We were unable to resolve specific endogenous Lrrk2 signals in WT (nTg) mice. In comparing mouse and human macrophages, we noticed mouse Lrrk2 was expressed at approximately one-fifth the level of human LRRK2 in the same number of cells cultured the same way, irrespective of IFN $\gamma$-induction or alternative polarization (Supplemental Fig. 5). Serendipitously, mouse cells overexpressing Lrrk2 from a mouse BAC insertion had similar levels of Lrrk2 protein as human cells, controlled from the endogenous Lrrk2 promoter (Supplemental Fig. 5). In the analysis of spleen cells from the WTLrrk2 BAC mice, we could successfully resolve Lrrk2signals in monocyte-enriched $\mathrm{CD} 45+/ \mathrm{CD} 11 \mathrm{~b}+$ populations (Fig. 4A).

To track possible Lrrk2-positive mouse immune cells in the brain after the intracranial injection of $\alpha$ synuclein fibrils, we performed flow cytometry with midbrain tissue homogenates from the WT-Lrrk2 BAC mice side-by-side with Lrrk2 $\mathrm{KO}$ mice as controls. The injection strategy and tissue dissected for analysis by flow cytometry is illustrated in Fig. 4B. Cell populations were gated according to CD11b and CD45 positivity (Fig. 4C, with gating strategy provided in Supplemental Fig. 6). Both Ly6C+ (enriched in classical monocytes according to [53]) and Ly6C- (enriched in non-classical monocytes) cell populations emerged in the $\mathrm{CD} 11 \mathrm{~b}+/ \mathrm{CD} 45^{\mathrm{hi}}$ population with robust Lrrk2 expression (Fig. 4D, E). In contrast, the much larger $\mathrm{CD} 11 \mathrm{~b}+/ \mathrm{CD} 45^{\mathrm{Lo}} / \mathrm{Ly} 6 \mathrm{C}$ - cell population (primarily microglia) had very low Lrrk2 or undetectable expression (Fig. 4E). The CD11b+/CD45 ${ }^{\text {hi }}$ population was miniscule with saline-only $72 \mathrm{~h}$ post injection (Supplemental Fig. 7), consistent with recent reports in rats suggesting saline or monomeric $\alpha$-synuclein injections do not result in the recruitment of peripheral immune cells to the brain [17]. No discernable signals were identified for Lrrk2 expression in the CD4 or CD8 T-cell populations that accumulated in the brain (Fig. 4E). After $72 \mathrm{~h}$ of intracranial injection of $\alpha$ synuclein fibrils, Lrrk2 levels between infiltrated and peripheral (blood) populations of $\mathrm{CD} 45^{\mathrm{hi}} / \mathrm{Ly} 6 \mathrm{C}+$ and CD $45^{\text {hi }} / \mathrm{Ly} 6 \mathrm{C}+$ were compared. The highest Lrrk2 levels we observed in this study localized to the CD45 hi Cd11b/Ly6C- cell compartment in the brain after fibril injection (Fig. 4E, F). Ly6C expression in CD45 $5^{\mathrm{hi}} / \mathrm{Cd} 11 \mathrm{~b} /$ Lrrk $2^{\text {hi }}$ cells in the brain may reflect a dynamic loss of Ly6C expression once Ly6 $C^{\text {hi }}$ monocytes commensurate with monocyte to macrophage maturation known to occur in tissues [54,55]. Alternatively, this could reflect a dynamic upregulation of Lrrk2 expression in nonclassic $\mathrm{CD} 45^{\mathrm{hi}} / \mathrm{Cd} 11 \mathrm{~b} / \mathrm{Ly} 6 \mathrm{C}$ - cells that remain Ly6C negative with maturation, or some combination of both processes.

\section{Mutant Lrrk2 expression increases pro-inflammatory monocyte recruitment to the brain shortly after a- synuclein fibril injection}

Previously, we found that intracranial injections of $\alpha$ synuclein fibrils into rat substantia nigra, but not monomeric protein or saline control, causes microglial activation and the upregulation of MHCII expression, as well as the recruitment of different proinflammatory immune cells to the brain [17]. Given our current observation that Lrrk2 expression appears inducible with $\alpha$-synuclein fibrils in the monocyte cell compartment, and our past work that suggests LRRK2 might control macrophage chemotaxis through Rab10 phosphorylation [9, 10], we sought to determine whether pathogenic LRRK2 mutations that upregulate LRRK2 kinase activity might affect 


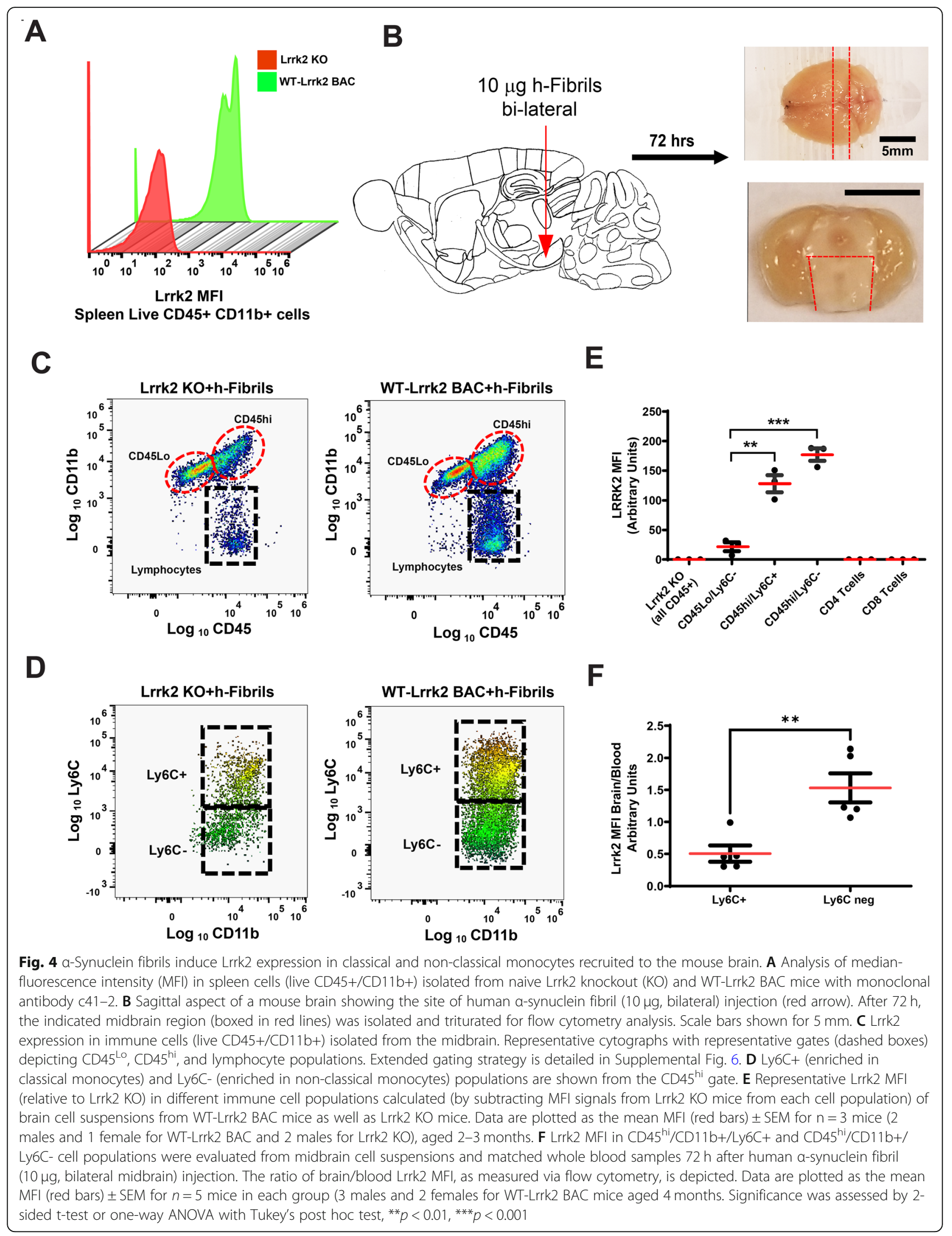


the recruitment of monocytes to the brain in response to $\alpha$-synuclein fibrils. We employed recently developed congenic (C57BL/6 J) R1441C-Lrrk2 knock-in (KI) mice as well as G2019S-BAC mice that show upregulated Lrrk2 expression and activity for in vivo chemotaxis experiments. In brain lysates from homozygous R1441C-KI mice, as well as G2019S-Lrrk2 BAC mice, we detected an increase of the Lrrk2 substrate pT73-Rab10 compared to their $\mathrm{WT}(\mathrm{nTg})$ littermate controls in naive animals (Fig. 5A, C). To measure immune cell responses in the brain after exposure to $\alpha$-synuclein fibrils, we performed flow cytometry analysis with tissue dissected according to Fig. 4B, $72 \mathrm{~h}$ after bi-lateral fibril injection. Gating strategies for these cohorts of mice are described in Supplemental Fig. 8. To account for the possible effects of surgery and the injection of saline, as well as the non-specific effects of the recombinant $\alpha$-synuclein protein, we first analyzed immune cell responses $72 \mathrm{~h}$ after monomer protein injection. With monomer only exposures, no differences were noted in the numbers of monocytes $\left(\mathrm{CD} 11 \mathrm{~b}+/ \mathrm{Ly} 6 \mathrm{G}-/ \mathrm{Ly} 6 \mathrm{C}^{\mathrm{hi}}\right)$ in the midbrain between WT(nTg) mice and R1441C-KI mice, though a slight suppression of the number of Ly6 $\mathrm{C}^{\mathrm{hi}}$ monocytes was noted in G2019S-Lrrk2 BAC mice midbrain homogenates (Fig. 5E). Thus, both lines of mutant Lrrk2 mice did not display basal differences in the brain with respect to monocyte numbers. However, with $\alpha$-synuclein fibril injections, we observed an increase in the number of CD11b+/Ly6G-/Ly6C $C^{\text {hi }}$ monocytes (i.e., proinflammatory infiltrating) in both R1441C-Lrrk2 KI (Fig. 5B) as well as G2019S-Lrrk2 BAC (Fig. 5D) mice when compared to their corresponding littermate WT(nTg) controls (Fig. 5F, G). No differences in the numbers of the more abundant microglia cell population was noted between the different strains (Supplemental Fig. 8), nor differences at this time point in the numbers of MHCII-positive activated microglia in the R1441CLrrk2 KI mice treated with monomers or fibrils (Fig. $5 \mathrm{H}$ ). These results suggest mutated LRRK2 may exacerbate the recruitment of pro-inflammatory monocyte-derived macrophages to the brain in response to pathogenic $\alpha$ synuclein.

\section{Lrrk2 kinase inhibition blocks monocyte-derived} macrophage chemotactic responses to a-synuclein fibrils Previously we and others found that $\alpha$-synuclein fibrils, but not monomeric protein, elicits the strong secretion of a number of murine chemokines and cytokines from microglia-like BV-2 cells, a mouse cell line with microglia features, including $\mathrm{Ccl} 5$ secretion known to stimulate monocyte chemotaxis $[17,56]$. LRRK2 kinase inhibition may attenuate chemotaxis in monocytederived macrophages, possibly through blocking Rab10 phosphorylation that boosts Ccl5-induced AKT activation [9]. We tested whether LRRK2 kinase inhibition might likewise have a direct effect on chemotactic responses in the context of WT or mutant Lrrk2 (R1441C-Lrrk2 KI or G2019S-Lrrk2 BAC) expression in mouse monocyte-derived macrophages (Fig. 6A). The application of conditioned media from $\alpha$-synuclein fibril-treated BV-2 cell cultures led to a $\sim 2$-fold increase in the number of macrophages migrating into the Boyden chamber matrix over $2 \mathrm{~h}$ (Fig. 6B, C). The addition of the LRRK2 kinase inhibitor MLi2 (100 nM) together with the $\alpha$-synuclein fibril conditioned media in the Boyden chamber blocked the increases in chemotaxis. In these cells, the effects of mutant Lrrk2 expression were less apparent, with the G2019S-Lrrk2 BAC cells showing increased numbers of migration into the matrix in response to the $\alpha$-synuclein fibril conditioned media (Fig. 6C). The $\alpha$-synuclein fibrils are not themselves chemoattractants, as exposures of equivalent concentrations of fibrils used to treat the BV-2 cells to the lower half of the Boyden chambers did not stimulate chemotaxis (Fig. 6D). Together, these results support a link between LRRK2 kinase activity and $\alpha$-synuclein fibril-induced chemotactic responses.

\section{Discussion}

In this study, we found that LRRK2 induction and activity in response to $\alpha$-synuclein fibrils is robust in monocyte-derived macrophages but largely absent in resident brain microglia in mice. LRRK2 induction via fibrilized $\alpha$-synuclein in cultured cells depends on JAKSTAT signaling as well as intrinsic LRRK2 kinase activity. These relationships appear conserved between mouse and human cultured immune cells, and between mouse and human strains of $\alpha$-synuclein fibrils, although lower levels of Lrrk2 are observed in mouse cells as compared to human cells. The mechanisms of LRRK2 induction observed here are largely consistent with prior studies that have shown that LRRK2 synergizes with interferon responses in iPSC-derived cells, and promotes LPS signaling in R1441G-transgenic mice [20, 26]. These results are also potentially consistent with previous studies suggesting LRRK2 kinase activity affects vesicle turnover and traffic in the endolysosomal system to strengthen chemokine signaling, possibly through a Rab10 and CCR5 dependent pathway [10]. Taken together, we hypothesize that LRRK2 drives neuroinflammatory responses in part by enhancing the recruitment of pro-inflammatory monocytes from the periphery. These processes may synergize with LRRK2 effects in the periphery that may also amplify pro-inflammatory responses to affect cells in the brain [26]. Following this hypothesis, future studies that specifically repress or activate LRRK2 only in the monocyte cell compartment in the context of disease may provide critical additional 


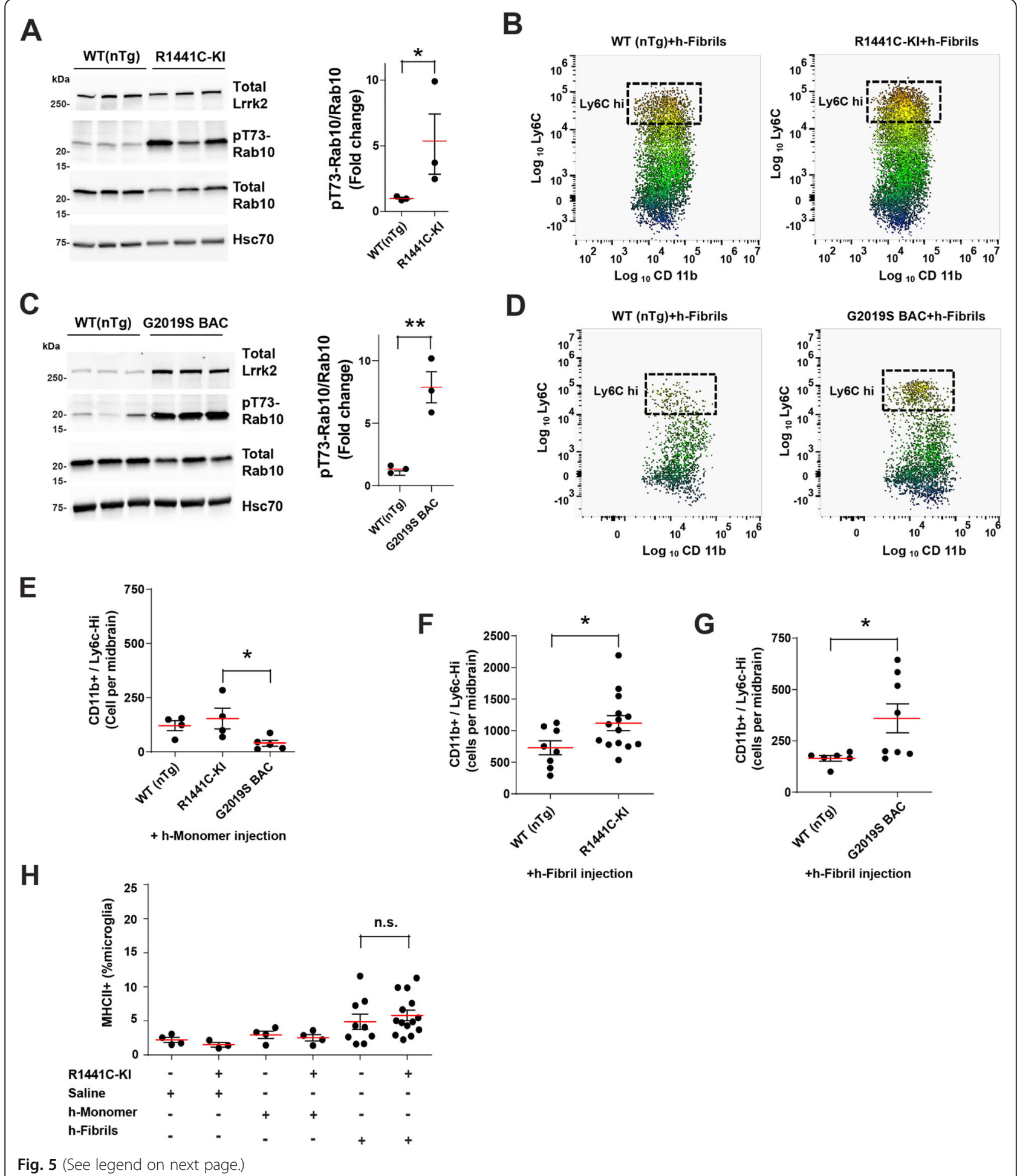


(See figure on previous page.)

Fig. 5 Increased a-synuclein fibril-induced infiltration of pro-inflammatory monocytes in Lrrk2 knock-in R1441C and G2019S-BAC mice. A Representative immunoblots and quantification of Lrrk2 expression and Rab10 phosphorylation (pT73-Rab10 to total Rab10) in brain lysates from WT (nTg) mice and homozygous Lrrk2-R1441C knock-in (KI) mice, and B live CD45 hi/CD11b+/Ly6Chi midbrain cells isolated from a mononuclearenriched Percoll gradient 3 days after injection (bilaterally) with $10 \mathrm{\mu g}$ of human a-synuclein fibrils (gating strategy is presented in Supplemental Fig. 8). C, D Mice expressing G2019S-Lrrk2 with the mutation knocked into a mouse BAC spanning the Lrrk2 gene were likewise analyzed together with littermate WT (nTg) controls. E Quantification of CD45 hi /CD11b+/Ly6C hi (enriched in pro-inflammatory classical monocytes) cells isolated from the brains of WT (nTg), Lrrk2-R1441C KI and G2019S BAC mice $72 \mathrm{~h}$ after the injection of $10 \mu \mathrm{g}$ (bilaterally) of monomeric human asynuclein. $\mathbf{F}$ In contrast to monomeric protein injections, the injection of fibrils ( $10 \mu \mathrm{g}$ bilateral, $72 \mathrm{~h}$ wait) results in the robust recruitment of CD45 hi $/ C D 11 \mathrm{~b}+/$ Ly6 $6 \mathrm{C}^{\text {hi }}$ cells in the mouse brain (PBS perfused and then analyzed by flow cytometry, see Methods) that is further increased in R1441C-KI mice and G G2019S-BAC mice. Microglia cell numbers after fibril treatment between strains are not different between groups (see Supplemental Fig. 8). $\mathbf{H}$ Percentage of activated microglia (CD45\%/CD11 b+/MHCII+) in WT (nTg) and R1441C-KI mice injected with saline, human a-synuclein monomers, or fibrils $(10 \mu \mathrm{g}$ bilateral) is shown in the graph. In each panel, each dot represents the analysis of an individual mouse with 13 mice ( 3 males and 10 females) analyzed in $\mathbf{E}, 22$ mice ( 9 males and 13 females) analyzed for $\mathbf{F}, 14$ mice (5 males and 9 females) analyzed for $\mathbf{G}$, and 37 mice (13 males and 24 females) analyzed for $\mathbf{H}$. All mice were aged 2-3 months at the time of the analysis. Data are plotted as the mean (red bars) \pm SEM. Significance is determined by unpaired $t$-tests ${ }^{*} p<0.05,{ }^{* *} p<0.01$

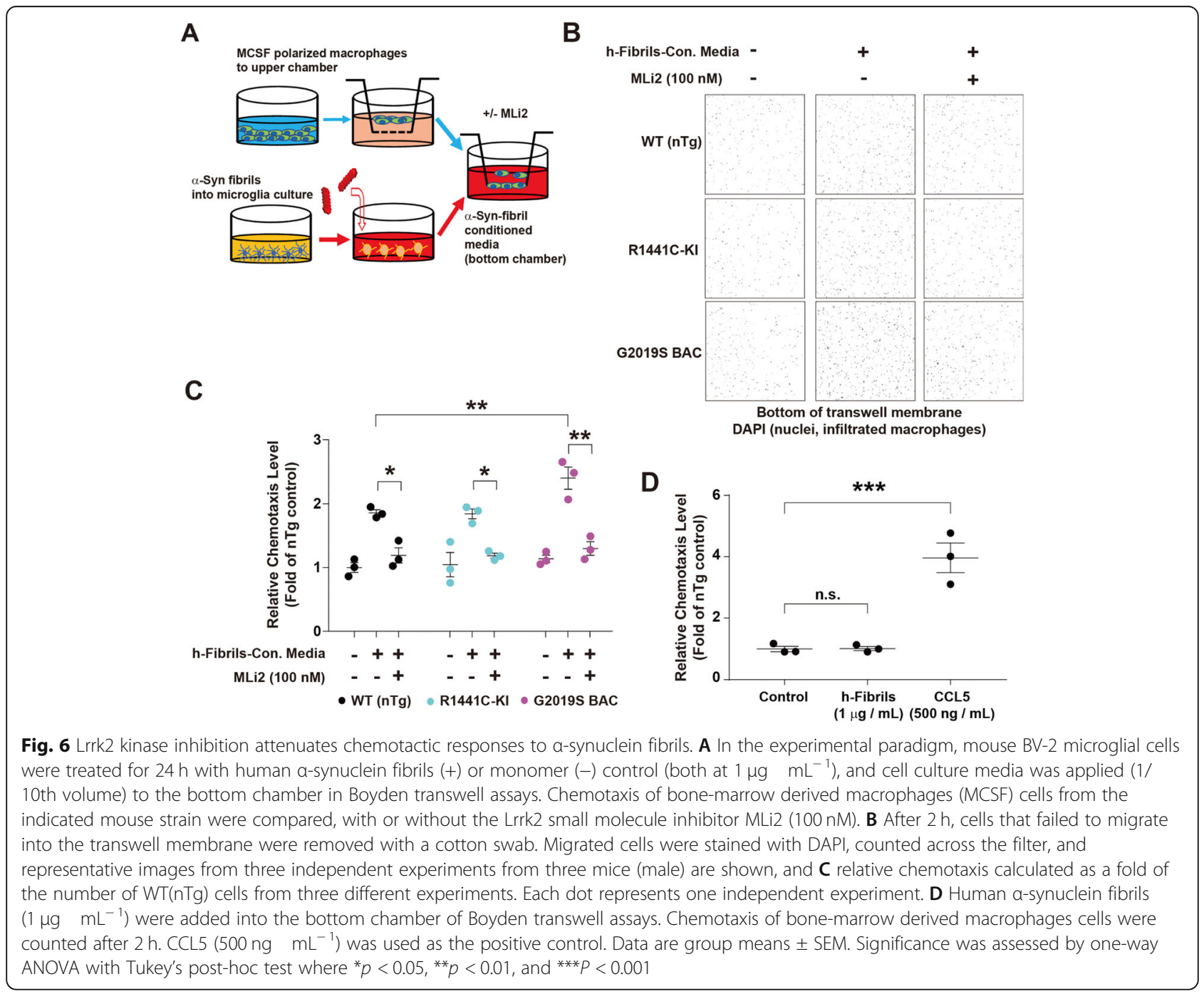


insights. A hypothesized model is proposed in Fig. 7, where chemoattractants from microglia, astrocytes, and other cells responsive to $\alpha$-synuclein may stimulate the recruitment of damaging pro-inflammatory LRRK2positive immune cells.

We found inhibition of JAK-STAT signaling to be a potent means of blocking $\alpha$-synuclein fibril induction of LRRK2 expression and activity. One of the JAK1/2 inhibitors used here, AZD1480, also provides protection against rAAV2- $\alpha$-synuclein induced neuroinflammation and dopaminergic neurodegeneration in the brain [33]. As LRRK2 inhibitors also may provide protection in a similar rAAV2- $\alpha$-synuclein model [57], we hypothesize that some of the neuroprotection afforded by AZD1480 may be through blocking LRRK2 induction in proinflammatory monocytes. Direct LRRK2 function and synergism in JAK-STAT signaling has yet to be fully clarified but may involve endocytosis, signaling endosome dynamics, and vesicle recycling. In intracellular analysis, the numbers of phospho-Rab10 vesicles were not increased with $\alpha$-synuclein-fibril stimulation. Instead, the amount of phospho-Rab10 on the vesicles was

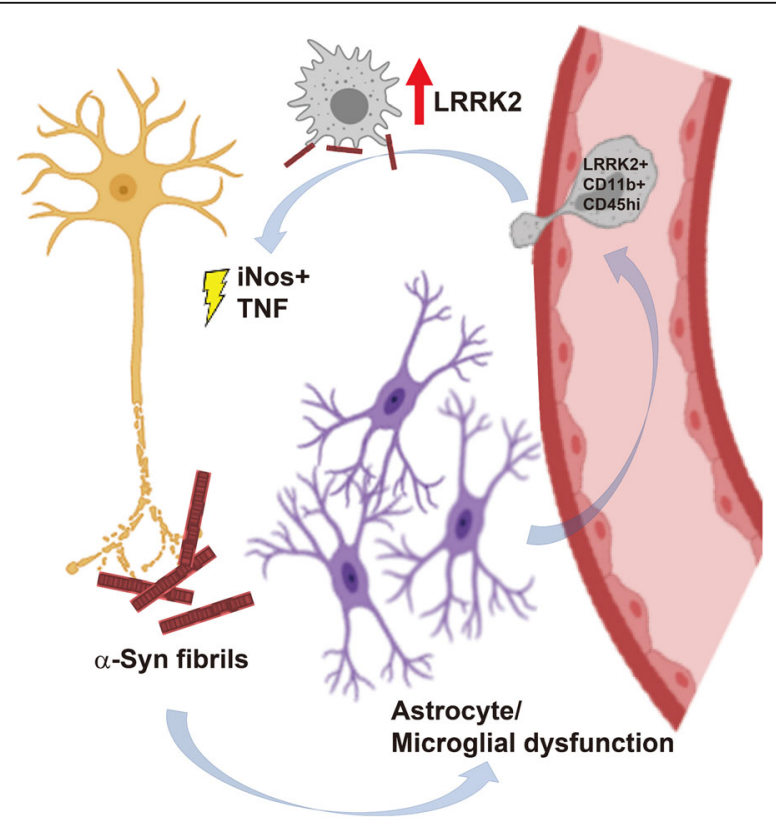

Fig. 7 Model for possible LRRK2 interaction with a-synuclein fibrils in neuroinflammation and neurodegeneration. Neuronal terminal damage, caused in part by the fibrillization and aggregation of asynuclein, may cause microglia and astrocyte responses that deleteriously affect the blood-brain barrier, the secretion of chemokine gradients, or possibly the release of aggregated asynuclein. Monocyte extravasation may occur with concomitant sensing of a-synuclein fibrils that elicit LRRK2 expression to facilitate chemotaxis into parenchymal tissues. Pro-inflammatory monocytes may control local production of damaging cytokines and oxidative responses to cause further neuronal damage in a feed-forward progressive pathway increased. These results may be in line with recent observations that show that phospho-Rab10 vesicles fail to recycle and thus persist in the cytosol, thereby strengthening signal transduction [9]. As opposed to more ubiquitous Rab substrates, LRRK2 is both inducible and highly restricted in expression across different immune cell subtypes (Supplemental Fig. 9). This may explain in part why the effect of LRRK2 on different immunological outcomes may be highly contextual and refined.

Based on past studies that demonstrate robust LRRK2 effects on microglial responses both in cell culture and in mouse models [19-22], it has been widely presumed (by us and others) that the intrinsic action of LRRK2 in microglia must drive immunological phenotypes. Yet, emergent single-cell and transcriptome wide sequencing databases have failed to identify appreciable Lrrk2 mRNA in mouse cells assigned to microglia lineages or brain-resident macrophage populations (Supplemental Fig. 9). Microglial cells in culture are known to acquire macrophage-like phenotypes distinct from authentic microglia in the mouse brain [58]. In a comparative analysis of cultured cells, we found that the cytokines and chemokines used to induce microglial-like states also suppress LRRK2 activation, but do not repress a robust response to $\alpha$-synuclein fibrils. How closely these artificially-directed cells mimic those that might occur in the brain and periphery in disease is not clear. Further, we cannot exclude the possibility of disease-state specific immune cell populations (e.g., rare T-cell subpopulations) that might rely on LRRK2 for appropriate signaling in disease. Future studies will be required to pinpoint how brain-resident pools of microglia might suppress Lrrk2 expression compared to monocytederived macrophage cells. We expect high-resolution deep single-nucleus sequencing efforts, particularly in patient cells and brain tissue samples, to help pinpoint brain-engrafting macrophages and rare subpopulations of cells that may be physiologically important.

In rodent models of $\mathrm{PD}$, peripheral immune cells interact with resident immune cells in diseased tissue [59]. Past studies implicate peripheral and systemic immunological states that can influence $\alpha$-synuclein fibrilinduced phenotypes in the brain [60]. In later time points (months) after fibril injection, but before dopaminergic neurodegeneration, recent reports have highlighted persistent infiltration of B cells, T-cells, and natural killer cells in the brain [16]. Increases in leukocyte subsets in the spleen and lymph nodes were also detected, although minimal changes in blood were noticed $\sim 5$ months' post-fibril injections. Based on our results, we would predict that early enhanced recruitment of pro-inflammatory cells to the brain might further exacerbate damaging systemic responses also mediated by LRRK2, with respect to dopaminergic 
neurodegeneration and the loss of other vulnerable neurons [26].

Further highlighting systemic responses in PD, two past reports suggest LRRK2 protein upregulation in human CD16+ blood monocytes using flow-cytometry $[7,8]$. Notably, the LRRK2 antibody utilized here (rabbit monoclonal [MJFF2 (c41-2)]) has similar affinity to both mouse and human LRRK2 in recognizing a conserved epitope $[8,25,61]$. Based on our results and those of others, we predict that upregulated LRRK2 expression and activity in blood monocytes may be associated with increased pro-inflammatory monocyte responses in the brain (Fig. 7). Whether or not these cells contribute to damaging cytokine and oxidative responses in the brain remains to be determined in future studies. A potential therapeutic strategy to address the effects of increased LRRK2 expression and activity in monocytes may be to utilize agents that mitigate monocyte infiltration to the brain. However, an alternative hypothesis for LRRK2 function in monocytes in disease may include LRRK2 regulation of circulating factors that may stimulate effects in the brain [26]. Further, such systemic effects may synergize with local immune cell recruitment to the brain in disease. Additional studies that help untangle these relationships could have important consequences for successful therapeutic approaches to mitigate LRRK2 activity.

In targeting pathways important for mounting immunological responses to different types of challenges, there could be adverse consequences with blocking LRRK2 in innate immune cells. Some of these issues have been discussed by others recently and in depth $[44,62]$. Future studies utilizing chemokine-receptor antagonists and other therapeutic candidates in the context of over-active mutant LRRK2 and $\alpha$-synuclein fibril-induced neurodegeneration may be informative. In macrophages, we recently identified an interaction between LRRK2 kinase activity and CCR5 traffic via Rab10 phosphorylation [9]. Although there is complicated redundancy in chemokine signaling pathways in vivo and more interactions that remain to be identified with respect to LRRK2 signaling, the inhibition of CCR5 on monocytes that express high LRRK2 levels may present an opportunity to mitigate the effects of mutant LRRK2 in the immune system.

While the effects of LRRK2 kinase inhibition on chemotaxis were unambiguous in cultured cells, we were unable to perform the same LRRK2 kinase inhibitor experiment in living mice with a full mammalian immune system. In rats, we and others have described acceptable pharmacokinetics and brain penetration with standard oral delivery for several structurally distinct LRRK2 inhibitors known to block Rab phosphorylation [35, 57, 63-65]. But in mice, we are not aware of a selective and brain penetrant LRRK2 inhibitor with a half-life greater than one-hour in oral dosing that might produce long- duration inhibition. In-diet dosing regimens have been successfully utilized in mice, harnessing the normal feeding behavior in mice to counteract the rapid turn-over and micromolar concentrations of drugs associated with off-target binding in oral, IP, or IV dosing [40]. However, feeding behavior is disrupted in many animal models of disease including the surgical procedure of intracranial injection of fibrils used in this study. In addition, even in the context of non-disrupted in-diet dosing in healthy mice, mutant G2019S LRRK2 appears resistant to full kinase inhibition with several inhibitor classes $[35,66]$. We further avoided observations in the global LRRK2 knockout model where compensatory mechanisms and lower white-blood cell counts may confound interpretations [67]. We predict that future studies that use genetic or transplantation methodologies to evaluate LRRK2 function in immune cells, as well as the evaluation of immune cell status in LRRK2 mutation carrier brain tissue, will be more informative in interpreting the function of LRRK2 in monocytes recruited to the brain.

\section{Conclusions}

Here we describe two new potential roles for LRRK2 function in monocyte-derived macrophages reacting to aggregated $\alpha$-synuclein. First, LRRK2 may facilitate initial chemotaxis in cells that express high LRRK2 levels, especially pro-inflammatory monocytes, that bring the cells into the brain parenchyma in response to the accumulation of aggregated $\alpha$-synuclein. Second, once exposed to aggregated $\alpha$-synuclein, JAK-STAT signaling may direct LRRK2 expression and kinase activity to bolster pro-inflammatory responses. Considering the role of LRRK2 kinase activity in enhancing ongoing interferon and NFATc2 signaling associated with damaging proinflammatory responses and chronic inflammation [20, 21], this process may have an overall detrimental effect on the survival of nearby vulnerable neurons. As LRRK2-targeted inhibitors enter into efficacy trials in both LRRK2 mutation carriers and idiopathic disease, our results place further emphasis on peripheral immune cells positive for LRRK2 expression in the disease process. We anticipate that part of the therapeutic benefit of LRRK2 inhibition, if realized in the clinic, may be through reducing pro-inflammatory responses of brain-engrafting monocyte-derived macrophages that accumulate over the course of the disease.

\section{Abbreviations}

LRRK2: Human leucine-rich repeat kinase 2; Lrrk2: Mouse leucine-rich repeat kinase 2; BMDM: Mouse bone marrow-derived macrophage; GMCS

F: Granulocyte macrophage colony stimulating factor;

LPS: Lipopolysaccharide; JAK: Janus kinase; MCSF: Macrophage colony

stimulating factor; MDMi: Monocyte-derived microglia-like cells;

PBMCs: Peripheral blood mononuclear cells; TLR: Toll-like receptor;

STAT: Signal transducer and activator of transcription 


\section{Supplementary Information}

The online version contains supplementary material available at https://doi. org/10.1186/s13024-021-00509-5.

\section{Additional file 1. \\ Additional file 2.}

Additional file 3

\section{Acknowledgements}

N/A

\section{Authors' contributions}

E.X., R.B., H.A., A.S., K.K., Z.L., N.B., performed experiments, analyzed data, and wrote the manuscript. S.C., E.L., E.B., A.H., E.G., A.W., analyzed data and wrote the manuscript. T.Y., L.V., and D.S. contributed critical tools and wrote the manuscript. The author(s) read and approved the final manuscript.

\section{Funding}

This work has been supported by NIH/NINDS P50-NS108675 (A.B.W, D.G.S, E.N.B, T.A.Y), NIH/NINDS R33-NS097643 (A.B.W), and NIH/NINDS R01NS064934 (A.B.W). Portions of this publication were made possible by the University of Alabama at Birmingham Center for Clinical and Translational Science Grant Number UL1-TR001417 from the National Center for Advancing Translational Sciences (NCATS) of the National Institutes of Health (NIH).

\section{Availability of data and materials}

The datasets used and/or analysed during the current study are available from the corresponding author on reasonable request.

\section{Declarations}

\section{Ethics approval and consent to participate}

All study protocols were approved by local Institutional Review Boards and Institutional Animal Care and Use Committees.

\section{Competing interests}

A.B.W. is a member of the Scientific Advisory Board for the Michael J. Fox Foundation and an active consultant for eScapeBio, Inc., and Neuro23, Inc., and has received research support unrelated to this study from Biogen/ldec. R.B. has no competing interests. K.K. has no competing interests. S.C. has no competing interests. S.M.C. has no competing interests. A.S. had no competing interests. D.G.S. is a member of the faculty of the University of Alabama at Birmingham and is supported by endowment and University funds. Dr. Standaert is an investigator in studies funded by Abbvie, Inc., the American Parkinson Disease Association, the Michael J. Fox Foundation for Parkinson Research, Alabama Department of Commerce, the Department of Defense, and NIH grants P50NS108675, R25NS079188, and T32NS095775. He has a clinical practice and is compensated for these activities through the University of Alabama Health Services Foundation. In addition, since January 1, 2020 he has served as a consultant for or received honoraria from Abbvie Inc., Sutter Health, the International Parkinson Disease and Movement Disorder Society, Theravance, McGraw Hill, Curium Pharma, F. Hoffmann LaRoche, Grey Matter Technologies and Sanofi-Aventis. E.N.B. is a member of the faculty of the University of Alabama at Birmingham and is supported by endowment and University funds. E.N.B. is an investigator in studies funded by the Michael J. Fox Foundation for Parkinson Research, the National Multiple Sclerosis Society, the Department of Defense, and NIH grants R01NS5756, R01-CA194414, P50-NS108675, R01NS1-9529, R01-CA246708, and R01-Al148711. E.N.B is also a consultant for or received honoraria from the SONTAG Brain Tumor Foundation, The Race to Erase MS Foundation, the $\mathrm{NIH}$, the American Association for Medical Colleges, Young Investigators Forum in Neuro-Oncology, Shanghai Jiantong University, and the Univ. of California at San Francisco.

\section{Author details}

'Duke Center for Neurodegeneration Research, Duke University, Durham, NC 27710, USA. ${ }^{2}$ Department of Pharmacology and Cancer Biology, Duke University, 3 Genome Court, Durham, NC 27710, USA ${ }^{3}$ Department of Neurology, University of lowa, lowa City, IA, USA. ${ }^{4}$ Medical Scientist Training
Program, Northwestern University Feinberg School of Medicine, Chicago, IL 60611, USA. ${ }^{5}$ Center for Clinical and Translational Science, University of Alabama at Birmingham, Birmingham, AL 35294, USA. ${ }^{6}$ Department of Chemistry and Biochemistry, New Mexico State University, Las Cruces, NM 88003, USA. ${ }^{7}$ Department of Microbiology, University of Alabama at Birmingham, Birmingham, AL 35294, USA. ${ }^{8}$ Center for Neurodegeneration and Experimental Therapeutics, Department of Neurology, University of Alabama at Birmingham, Birmingham, AL 35216, USA. 'Department of Cell, Developmental and Integrative Biology, University of Alabama at Birmingham, Birmingham, AL 35294, USA.

Received: 27 May 2021 Accepted: 14 December 2021

Published online: 10 January 2022

\section{References}

1. Trinh J, Guella I, Farrer MJ. Disease penetrance of late-onset parkinsonism: a meta-analysis. JAMA Neurol. 2014;71:1535-9.

2. West $A B$. Achieving neuroprotection with LRRK2 kinase inhibitors in Parkinson disease. Exp Neurol. 2017;298:236-45.

3. Nalls MA, Blauwendraat C, Vallerga CL, Heilbron K, Bandres-Ciga S, Chang D, et al. Identification of novel risk loci, causal insights, and heritable risk for Parkinson's disease: a meta-analysis of genome-wide association studies. Lancet Neurol. 2019;18:1091-102.

4. Foo JN, Chew EGY, Chung SJ, Peng R, Blauwendraat C, Nalls MA, et al. Identification of risk loci for Parkinson disease in Asians and comparison of risk between Asians and Europeans: a genome-wide association study. JAMA Neurol. 2020;77:746-54.

5. Pierce S, Coetzee GA. Parkinson's disease-associated genetic variation is linked to quantitative expression of inflammatory genes. PLoS One. 2017;12: e0175882.

6. Raj T, Rothamel K, Mostafavi S, Ye C, Lee MN, Replogle JM, et al. Polarization of the effects of autoimmune and neurodegenerative risk alleles in leukocytes. Science. 2014:344:519-23.

7. Bliederhaeuser C, Zondler L, Grozdanov V, Ruf WP, Brenner D, Melrose HL, et al. LRRK2 contributes to monocyte dysregulation in Parkinson's disease. Acta Neuropathol Commun. 2016; Available from: https://doi.org/10.1186/s4 0478-016-0396-2.

8. Cook DA, Kannarkat GT, Cintron AF, Butkovich LM, Fraser KB, Chang J, et al. LRRK2 levels in immune cells are increased in Parkinson's disease. NPJ Parkinsons Dis. 2017:3:11

9. Liu Z, Xu E, Zhao HT, Cole T, West AB. LRRK2 and Rab10 coordinate macropinocytosis to mediate immunological responses in phagocytes. EMBO J. 2020;39:e104862.

10. Moehle MS, Daher JPL, Hull TD, Boddu R, Abdelmotilib HA, Mobley J, et al. The G2019S LRRK2 mutation increases myeloid cell chemotactic responses and enhances LRRK2 binding to actin-regulatory proteins. Hum Mol Genet. 2015;24:4250-67.

11. Levy DR, Udgata A, Tourlomousis P, Symmons MF, Hopkins $\sqcup$, Bryant CE, et al. The Parkinson's disease-associated kinase LRRK2 regulates genes required for cell adhesion, polarization, and chemotaxis in activated murine macrophages. J Biol Chem. 2020:10857-10867. Available from: https://doi. org/10.1074/jbc.ra119.011842

12. Mir R, Tonelli F, Lis P, Macartney T, Polinski NK, Martinez TN, et al. The Parkinson's disease VPS35[D620N] mutation enhances LRRK2-mediated Rab protein phosphorylation in mouse and human. Biochem J. 2018:475:186183

13. Harms AS, Thome AD, Yan Z, Schonhoff AM, Williams GP, Li X, et al. Peripheral monocyte entry is required for alpha-Synuclein induced inflammation and Neurodegeneration in a model of Parkinson disease. Exp Neurol. 2018:179-87 Available from: https://doi.org/10.1016/j.expneurol.201 7.11.010.

14. Baufeld C, O'Loughlin E, Calcagno N, Madore C, Butovsky O. Differential contribution of microglia and monocytes in neurodegenerative diseases. J Neural Transm. 2018;125:809-26.

15. Daher JPL, Volpicelli-Daley LA, Blackburn JP, Moehle MS, West AB. Abrogation of a-synuclein-mediated dopaminergic neurodegeneration in LRRK2-deficient rats. Proc Natl Acad Sci U S A. 2014;111:9289-94.

16. Earls RH, Menees KB, Chung J, Barber J, Gutekunst C-A, Hazim MG, et al. Intrastriatal injection of preformed alpha-synuclein fibrils alters central and peripheral immune cell profiles in non-transgenic mice. J Neuroinflammation. 2019;16:250. 
17. Harms AS, Delic V, Thome AD, Bryant N, Liu Z, Chandra S, et al. a-Synuclein fibrils recruit peripheral immune cells in the rat brain prior to neurodegeneration. Acta Neuropathol Commun. 2017;5:85.

18. Goldmann T, Wieghofer P, Jordão MJC, Prutek F, Hagemeyer N, Frenzel K, et al. Origin, fate and dynamics of macrophages at central nervous system interfaces. Nat Immunol. 2016;17:797-805.

19. Moehle MS, Webber PJ, Tse T, Sukar N, Standaert DG, DeSilva TM, et al. LRRK2 inhibition attenuates microglial inflammatory responses. J Neurosci. 2012;32:1602-11.

20. Panagiotakopoulou V, Ivanyuk D, De Cicco S, Haq W, Arsić A, Yu C, et al. Interferon- $\gamma$ signaling synergizes with LRRK2 in neurons and microglia derived from human induced pluripotent stem cells. Nat Commun. 2020;11:5163.

21. Kim C, Beilina A, Smith N, Li Y, Kim M, Kumaran R, et al. LRRK2 mediates microglial neurotoxicity via NFATc2 in rodent models of synucleinopathies. Sci Transl Med. 2020;12 Available from: https://doi.org/10.1126/scitranslmed.aay0399.

22. Dwyer Z, Rudyk C, Thompson A, Farmer K, Fenner B, Fortin T, et al. Leucinerich repeat kinase-2 (LRRK2) modulates microglial phenotype and dopaminergic neurodegeneration. Neurobiol Aging. 2020;91:45-55.

23. Zhang Y, Chen K, Sloan SA, Bennett ML, Scholze AR, O'Keeffe S, et al. An RNA-sequencing transcriptome and splicing database of glia, neurons, and vascular cells of the cerebral cortex. J Neurosci. 2014;34:11929-47.

24. Saunders A, Macosko EZ, Wysoker A, Goldman M, Krienen FM, de Rivera H, et al. Molecular diversity and specializations among the cells of the adult mouse brain. Cell. 2018;174:1015-30.e16.

25. Davies P, Hinkle KM, Sukar NN, Sepulveda B, Mesias R, Serrano G, et al. Comprehensive characterization and optimization of anti-LRRK2 (leucinerich repeat kinase 2) monoclonal antibodies. Biochem J. 2013;453:101-13.

26. Kozina E, Sadasivan S, Jiao Y, Dou Y, Ma Z, Tan H, et al. Mutant LRRK2 mediates peripheral and central immune responses leading to neurodegeneration in vivo. Brain. 2018;141:1753-69.

27. Russo I, Kaganovich A, Ding J, Landeck N, Mamais A, Varanita T, et al. Transcriptome analysis of LRRK2 knock-out microglia cells reveals alterations of inflammatory- and oxidative stress-related pathways upon treatment with a-synuclein fibrils. Neurobiol Dis. 2019;129:67-78.

28. Dzamko N, Inesta-Vaquera F, Zhang J, Xie C, Cai H, Arthur S, et al. The IkappaB kinase family phosphorylates the Parkinson's disease kinase LRRK2 at Ser935 and Ser910 during toll-like receptor signaling. PLoS One. 2012;7: e39132.

29. Ryan KJ, White CC, Patel K, Xu J, Olah M, Replogle JM, et al. A human microglia-like cellular model for assessing the effects of neurodegenerative disease gene variants. Sci Transl Med. 2017;9 Available from: https://doi. org/10.1126/scitranslmed.aai7635.

30. Horvath RJ, Nutile-McMenemy N, Alkaitis MS, Deleo JA. Differential migration, LPS-induced cytokine, chemokine, and NO expression in immortalized BV-2 and HAPI cell lines and primary microglial cultures. J Neurochem. 2008;107:557-69.

31. Sokratian A, Ziaee J, Kelly K, Chang A, Bryant N, Wang S, et al. Heterogeneity in a-synuclein fibril activity correlates to disease phenotypes in Lewy body dementia. Acta Neuropathol. 2021;141:547-64.

32. Luk KC, Kehm V, Carroll J, Zhang B, O'Brien P, Trojanowski JQ, et al. Pathological a-synuclein transmission initiates Parkinson-like neurodegeneration in nontransgenic mice. Science. 2012;338:949-53.

33. Qin H, Buckley JA, Li X, Liu Y, Fox TH, Meares GP, et al. Inhibition of the JAK STAT pathway protects against a-synuclein-induced neuroinflammation and dopaminergic neurodegeneration. J Neurosci. 2016:5144-59 Available from: https://doi.org/10.1523/jneurosci.4658-15.2016.

34. Lever JM, Hull TD, Boddu R, Pepin ME, Black LM, Adedoyin OO, et al. Resident macrophages reprogram toward a developmental state after acute kidney injury. JCl Insight. 2019;4 Available from: https://doi.org/10.1172/jci. insight.125503.

35. Kelly K, Wang S, Boddu R, Liu Z, Moukha-Chafiq O, Augelli-Szafran C, et al. The G2019S mutation in LRRK2 imparts resiliency to kinase inhibition. Exp Neurol. 2018;309:1-13.

36. Trinkaus VA, Riera-Tur I, Martínez-Sánchez A, Bäuerlein FJB, Guo Q, Arzberger T, et al. In situ architecture of neuronal a-Synuclein inclusions. Nat Commun. 2021;12:2110.

37. Roy S, Wolman L. Ultrastructural observations in Parkinsonism. J Pathol. 1969;99:39-44.

38. Volpicelli-Daley LA, Luk KC, Patel TP, Tanik SA, Riddle DM, Stieber A, et al. Exogenous a-synuclein fibrils induce Lewy body pathology leading to synaptic dysfunction and neuron death. Neuron. 2011;72:57-71.
39. Abdelmotilib H, Maltbie T, Delic V, Liu Z, Hu X, Fraser KB, et al. a-Synuclein fibril-induced inclusion spread in rats and mice correlates with dopaminergic Neurodegeneration. Neurobiol Dis. 2017;105:84-98.

40. Fell MJ, Mirescu C, Basu K, Cheewatrakoolpong B, DeMong DE, Ellis JM, et al MLi-2, a potent, selective, and centrally active compound for exploring the therapeutic potential and safety of LRRK2 kinase inhibition. J Pharmacol Exp Ther. 2015:355:397-409.

41. Volpicelli-Daley LA, Abdelmotilib H, Liu Z, Stoyka L, Daher JPL, Milnerwood AJ, et al. G2019S-LRRK2 expression augments a-Synuclein sequestration into inclusions in neurons. J Neurosci. 2016;36:7415-27.

42. Hughes CD, Choi ML, Ryten M, Hopkins L, Drews A, Botía JA, et al. Picomolar concentrations of oligomeric alpha-synuclein sensitizes TLR4 to play an initiating role in Parkinson's disease pathogenesis. Acta Neuropathol. 2019;137:103-20

43. Venezia S, Refolo V, Polissidis A, Stefanis L, Wenning GK, Stefanova N. Tolllike receptor 4 stimulation with monophosphoryl lipid a ameliorates motor deficits and nigral neurodegeneration triggered by extraneuronal asynucleinopathy. Mol Neurodegener. 2017;12:52

44. Ahmadi Rastegar D, Dzamko N. Leucine rich repeat kinase 2 and innate immunity. Front Neurosci. 2020;14:193.

45. Quintás-Cardama A, Vaddi K, Liu P, Manshouri T, Li J, Scherle PA, et al. Preclinical characterization of the selective JAK1/2 inhibitor INCB018424: therapeutic implications for the treatment of myeloproliferative neoplasms. Blood. 2010;115:3109-17.

46. Derenzini $E$, Lemoine $M$, Buglio D, Katayama $H$, Ji Y, Davis RE, et al. The JAK inhibitor AZD1480 regulates proliferation and immunity in Hodgkin lymphoma. Blood Cancer J. 2011;1:e46.

47. Flanagan ME, Blumenkopf TA, Brissette WH, Brown MF, Casavant JM, ShangPoa C, et al. Discovery of CP-690,550: a potent and selective Janus kinase (JAK) inhibitor for the treatment of autoimmune diseases and organ transplant rejection. J Med Chem. 2010;53:8468-84.

48. Changelian PS, Flanagan ME, Ball DJ, Kent CR, Magnuson KS, Martin WH, et al. Prevention of organ allograft rejection by a specific Janus kinase 3 inhibitor. Science. 2003;302:875-8.

49. Fan Y, Howden AJM, Sarhan AR, Lis P, Ito G, Martinez TN, et al. Interrogating Parkinson's disease LRRK2 kinase pathway activity by assessing Rab10 phosphorylation in human neutrophils. Biochem J. 2018;475:23-44.

50. McGeer PL, Itagaki S, Boyes BE, McGeer EG. Reactive microglia are positive for HLA-DR in the substantia nigra of Parkinson's and Alzheimer's disease brains. Neurology. 1988:38:1285-91.

51. Imamura K, Hishikawa N, Sawada M, Nagatsu T, Yoshida M, Hashizume Y. Distribution of major histocompatibility complex class II-positive microglia and cytokine profile of Parkinson's disease brains. Acta Neuropathol. 2003; 106:518-26.

52. Sanchez-Guajardo V, Febbraro F, Kirik D, Romero-Ramos M. Microglia acquire distinct activation profiles depending on the degree of alphasynuclein neuropathology in a rAAV based model of Parkinson's disease. PLoS One. 2010;5:e8784.

53. Yona S, Kim K-W, Wolf Y, Mildner A, Varol D, Breker M, et al. Fate mapping reveals origins and dynamics of monocytes and tissue macrophages under homeostasis. Immunity. 2013;38:79-91.

54. Graubardt N, Vugman M, Mouhadeb O, Caliari G, Pasmanik-Chor M, Reuveni D, et al. Ly6C monocytes and their macrophage descendants regulate neutrophil function and clearance in acetaminophen-induced liver injury. Front Immunol. 2017:8:626.

55. Menezes S, Melandri D, Anselmi G, Perchet T, Loschko J, Dubrot J, et al. The heterogeneity of Ly6C monocytes controls their differentiation into iNOS macrophages or monocyte-derived dendritic cells. Immunity. 2016;45:1205-18.

56. Grozdanov V, Bousset L, Hoffmeister M, Bliederhaeuser C, Meier C, Madiona $K$, et al. Increased immune activation by pathologic a-synuclein in Parkinson's disease. Ann Neurol. 2019;86:593-606.

57. Daher JPL, Abdelmotilib HA, Hu X, Volpicelli-Daley LA, Moehle MS, Fraser KB, et al. Leucine-rich repeat kinase 2 (LRRK2) pharmacological inhibition abates a-synuclein gene-induced neurodegeneration. J Biol Chem. 2015;290: 19433-44.

58. Butovsky O, Jedrychowski MP, Moore CS, Cialic R, Lanser AJ, Gabriely G, et al. Identification of a unique TGF- $\beta$-dependent molecular and functional signature in microglia. Nat Neurosci. 2014;17:131-43.

59. Tansey MG, Romero-Ramos M. Immune system responses in Parkinson's disease: early and dynamic. Eur J Neurosci. 2018; Available from: https://doi. org/10.1111/ejn.14290. 
60. Peralta Ramos JM, Iribarren $P$, Bousset $L$, Melki R, Baekelandt V, Van der Perren A. Peripheral inflammation regulates CNS immune surveillance through the recruitment of inflammatory monocytes upon systemic asynuclein administration. Front Immunol. 2019:10:80.

61. West AB, Cowell RM, Daher JPL, Moehle MS, Hinkle KM, Melrose HL, et al. Differential LRRK2 expression in the cortex, striatum, and substantia nigra in transgenic and nontransgenic rodents. J Comp Neurol. 2014;522:2465-80.

62. Herrick MK, Tansey MG. Is LRRK2 the missing link between inflammatory bowel disease and Parkinson's disease? NPJ Parkinsons Dis. 2021;7:26.

63. Kelly K, Chang A, Hastings L, Abdelmotilib H, West AB. Genetic background influences LRRK2-mediated Rab phosphorylation in the rat brain. Brain Res. 2021;1759:147372

64. Kelly K, West AB. Pharmacodynamic biomarkers for emerging LRRK2 therapeutics. Front Neurosci. 2020;14:807.

65. Nguyen APT, Tsika E, Kelly K, Levine N, Chen X, West AB, et al.

Dopaminergic neurodegeneration induced by Parkinson's disease-linked G2019S LRRK2 is dependent on kinase and GTPase activity. Proc Natl Acad Sci U S A. 2020;117:17296-307.

66. Henderson MX, Peng C, Trojanowski JQ, Lee VMY. LRRK2 activity does not dramatically alter a-synuclein pathology in primary neurons. Acta Neuropathol Commun. 2018;6:45.

67. Baptista MAS, Dave KD, Frasier MA, Sherer TB, Greeley M, Beck MJ, et al. Loss of leucine-rich repeat kinase 2 (LRRK2) in rats leads to progressive abnormal phenotypes in peripheral organs. PLoS One. 2013;8:e80705.

\section{Publisher's Note}

Springer Nature remains neutral with regard to jurisdictional claims in published maps and institutional affiliations.

Ready to submit your research? Choose BMC and benefit from:

- fast, convenient online submission

- thorough peer review by experienced researchers in your field

- rapid publication on acceptance

- support for research data, including large and complex data types

- gold Open Access which fosters wider collaboration and increased citations

- maximum visibility for your research: over $100 \mathrm{M}$ website views per year

At $\mathrm{BMC}$, research is always in progress.

Learn more biomedcentral.com/submissions 